Confidential resubmission 07-28-2016

IB-D-16-00134R

For: Insect Biochemistry and Molecular Biology

\title{
Molecular and functional characterization of Bemisia tabaci aquaporins reveals the water channel diversity of hemipteran insects
}

Evelien Van Ekert ${ }^{\mathrm{a}}$, François Chauvigné ${ }^{\mathrm{b}, \mathrm{c}}$ Roderick Nigel Finn $^{\mathrm{b}, \mathrm{d}}$, Lolita G. Mathew ${ }^{\mathrm{a}}$, J. Joe Hull $^{\mathrm{a}}$, Joan Cerdà ${ }^{\mathrm{c}}$, Jeffrey A. Fabrick ${ }^{\mathrm{a}}{ }^{*}$

${ }^{a}$ USDA-ARS U.S. Arid Land Agricultural Research Center, Maricopa, AZ, 85138

${ }^{b}$ Department of Biology, Bergen High Technology Centre, University of Bergen, 5020 Bergen, Norway

${ }^{c}$ Institut de Recerca i Tecnologia Agroalimentaries (IRTA)-Institut de Ciencies del Mar, Consejo Superior de Investigaciones Cientificas (CSIC), 08003 Barcelona, Spain

d Institute of Marine Research, Nordnes, 5817 Bergen, Norway

*Corresponding author:

J.A. Fabrick

USDA-ARS Arid Land Agricultural Center, Maricopa AZ, 85138, USA

Tel: +1-520-316-6335

Fax: +1-520-316-6330

Email: jeff.fabrick@ars.usda.gov 


\section{Abstract}

The Middle East-Asia Minor 1 (MEAM1) whitefly, Bemisia tabaci (Gennadius) is an economically important pest of food, fiber, and ornamental crops. This pest has evolved a number of adaptations to overcome physiological challenges, including 1) the ability to regulate osmotic stress between gut lumen and hemolymph after imbibing large quantities of a low nitrogen, sugar-rich liquid diet; 2) the ability to avoid or prevent dehydration and desiccation, particularly during egg hatching and molting; and 3) to be adapted for survival at elevated temperatures. One superfamily of proteins involved in the maintenance of fluid homeostasis in many organisms includes the aquaporins, which are integral membrane channel proteins that aid in the rapid flux of water and other small solutes across biological membranes. Here, we show that B. tabaci has eight aquaporins (BtAqps), of which seven belong to the classical aquaporin 4-related grade of channels, including Bib, Drip, Prip, and Eglps and one that belongs to the unorthodox grade of aquaporin 12-like channels. B. tabaci has further expanded its repertoire of water channels through the expression of three BtDrip2 amino-terminal splice variants, while other hemipteran species express amino- or carboxyl-terminal isoforms of Drip, Prip, and Eglps. Each BtAqp has unique transcript expression profiles, cellular localization, and/or substrate preference. Our phylogenetic and functional data reveal that hemipteran insects lost the classical glp genes, but have compensated for this by duplicating the eglp genes early in their evolution to comprise at least three separate clades of glycerol transporters.

Keywords: Bemisia tabaci, whitefly, osmoregulation, aquaporin, entomoglyceroporin, major intrinsic protein family 


\title{
Highlights
}

- $\quad$ Eight unique aquaporins are present in the Middle East-Asia Minor 1 (MEAM1) cryptic species of Bemisia tabaci.

- The BtAqps belong to six phylogenetically distinct groups of arthropod aquaporins, including Bibs, Drips, Prips, EglpAs, EglpBs, and Aqp12Ls.

- Hemipteran insects lost the classical glp genes associated with glycerol transport, but compensated for this loss by evolving multiple forms of the Eglp channels.

\begin{abstract}
Abbreviations
Aqp, aquaporin; ar/R, aromatic arginine; Bib, big brain; BtAqp, Bemisia tabaci aquaporin; cDNA, complementary DNA; CDS, coding sequence; cRNA, complementary RNA; Drip, Drosophila integral protein; EGFP, enhanced green fluorescent protein; Eglp, entomoglyceroporin; ER, endoplasmic reticulum; EST, expressed sequence tag; Glp; aquaglyceroporin; hAQP, human aquaporin; MEAM1, Middle East-Asia Minor 1; MBS, modified Barth's media; MW, molecular weight; NPA motif, asparagine-prolinealanine motif; ORF, open reading frame; PCR, polymerase chain reaction; $\mathrm{pI}$, isoelectric point; PMSF, phenylmethylsulfonyl fluoride; Prip, Pyrocoelia rufa integral protein; RACE, rapid amplification of cDNA ends; RT-PCR, reverse transcriptase polymerase chain reaction; SRA, Short Read Archive; TM, transmembrane.
\end{abstract}

\section{Introduction}

Aquaporins belong to the major intrinsic protein family and function as bidirectional transport channels of water or other small solutes across biological membranes (Benga, 2009; Campbell et al., 2008; Murata et al., 2000; King et al., 2004; Gomes et al., 2009). Aquaporins are widely distributed in all kingdoms of life (Abascal et al., 2014; Finn and Cerdà, 2015) and play numerous essential physiological roles, particularly in regulating cell water balance. Generally, aquaporins consist of six transmembrane (TM) alpha 
helices connected through five loops (A-E) in an "hourglass" form (Jung et al., 1994). The amino- and carboxyl-termini are located on the cytoplasmic side of the membrane and each channel consists of two similar halves formed by a tandem repeat. Loops B (cytoplasmic) and E (extracellular) both contain the signature Asn, Pro, Ala (NPA) motif, and these two loops fold back into the channel from opposite sides of the membrane to form a seventh "broken" half membrane helix with the NPA motif at the center of the channel (Agre et al., 1993; Finn and Cerdà, 2015; Sui et al., 2001; Törnroth-Horsefield, 2010; Zardoya, 2005). Solute selectivity is determined by the constriction region at the extracellular side of the channel formed by an aromatic Arg (ar/R) selectivity filter (Beitz et al., 2006; Gonen and Walz, 2006; Jung et al. 1994). While each monomeric aquaporin can independently function as an individual water pore, they normally oligomerize as tetramers in the cell membrane forming a central pore, which in some cases can function as an ion channel (Agre et al., 1993; Gomes et al., 2009; Yool and Weinstein, 2002).

Eukaryotic aquaporins are divided into four major grades, including the classical aquaporins, the aquaporin 8-type aquaamoniaporins, the aquaporin 11/aquaporin 12-type unorthodox channels, and the aquaglyceroporins (Glps) (Finn and Cerdà, 2015). Previous attempts to classify the increasing number of arthropod aquaporins have provided several somewhat ambiguous groupings, including the identification of three subfamilies (Campbell et al., 2008), four groups (Kambara et al., 2009; Goto et al., 2011), or two to five clades (Wallace et al., 2012; Fabrick et al., 2014; Jing et al., 2016). More recent classification suggests that insects have aquaporins belonging to three major grades, including the classical aquaporins, the Glps, and the unorthodox aquaporin 12-like (Aqp12L) proteins (Finn et al., 2015; Stavang et al., 2015). The classical arthropod aquaporins include genes previously characterized as Clade A of the classical insect aquaporins (Wallace et al., 2012; Fabrick et al., 2014; Jing et al., 2016) and encompass 
four major subfamilies, including the Drosophila integral protein (Drip)-like aquaporins (Kaufmann et al., 2005), the Pyrocoelia rufa integral protein (Prip)-like aquaporins (Lee et al., 2001), the big brain proteins (Bib) (Campbell et al., 2008) and the recently described entomoglyceroporins (Eglps) (Finn et al., 2015). These Eglps, which can transport water, urea, and a range of polyols including glycerol, evolved through mutation of the conserved His in the ar/R selectivity filter of water-selective channels and are phylogenetically more closely related to the classical aquaporin 4-type channels than to the Glps (Finn et al., 2015). Furthermore, while the Glps are prevalent in the older lineages of hexapods, several modern lineages, including the holometabolous insects, appear to have lost Glps and alternatively possess expanded clusters of eglp genes (Finn et al., 2015).

Due to increased availability of sequencing data from insects, a number of unorthodox Aqp12L aquaporins are now being identified (Fabrick et al., 2014; Finn et al., 2015; Stavang et al., 2015). The Aqp12L channels are distinguished by the fact that they are not transported to the plasma membrane when expressed in Xenopus laevis oocytes, contain a non-canonical NPC motif, and the Arg in the ar/R selectivity filter is replaced by a Leu (Morishita et al., 2005; Gorelick et al., 2006, Finn and Cerdà, 2015). The actual function of such Aqp12L channels in insects and other arthropods remains unknown.

Insect aquaporins are involved in many physiological processes, including some that enable insects to process large volumes of liquid diet and overcome extreme conditions such as temperature, osmotic pressure due to sugar-rich diets, and desiccation (Campbell et al., 2008; Cohen, 2012, 2013; Spring et al., 2009). Therefore, they have potential as novel targets for pest control (Douglas, 2006; Cohen, 2013). One of the most common and costliest pests of the agricultural world is the Middle East-Asia Minor 1 (MEAM1) whitefly, Bemisia tabaci (Gennadius) (Hemiptera: Aleyrodidae) (hereafter denoted as B. 
tabaci). This pest harms crops by feeding on large quantities of phloem sap and excreting copious amounts of honeydew, which reduces postharvest quality (e.g. "sticky cotton") and promotes growth of sooty mold fungi, and by transmitting pathogenic viruses, such as the agriculturally important Begomoviruses (Byrne and Bellows 1991, Navas-Castillo et al., 2011).

Because B. tabaci ingests large volumes of sugar-rich, amino acid deficient phloem sap (Douglas, 2006), the high simple sugar content of phloem results in osmotic stress placed on the tissues of the alimentary system. Whiteflies possess both biochemical and physiological mechanisms to help alleviate the problem of both large quantities of liquid diet and osmotic stress. Enzymatic transformation of sugars from high to lower osmotic potential is thought to reduce osmotic potential in the whitefly gut (Salvucci et al., 2003). Whiteflies also use a filter chamber to transfer water between the proximal and distal gut regions (Goodchild, 1966; Cicero et al., 1995; Douglas, 2006; Hubert et al. 1989) that likely enables rapid removal of excess fluid and helps decrease osmotic potential. We previously demonstrated that a $B$. tabaci aquaporin (referred to as BtAQP1) functions as a water-specific Drip channel that is highly expressed in the filter chamber (Mathew et al. 2011). Here, we provide molecular and functional characterization of eight $B$. tabaci aquaporins (including the previously described BtDrip1).

Using the classification system of Finn and Cerdà (2015), we show that whiteflies and other hemipterans lack classical Glps and have evolved multiple eglp genes. B. tabaci has an extended repertoire of three Eglps, which together with two Drips, a Prip, and a Bib comprise the B. tabaci classical aquaporins. B. tabaci also contains one unorthodox Aqp12L. Functional oocyte swelling assays show specificity of the BtAqps for water and glycerol, and together with expression profiling and cellular localization, the potential importance of the superfamily for nutrient and water homeostasis. 


\section{Materials and methods}

\subsection{Insects}

A B. tabaci colony was maintained on broccoli (Brassica oleracea, Italica Group) inside a $0.9 \times 0.9 \times 2 \mathrm{~m}^{3}$ cage in a greenhouse (maintained between $21-32{ }^{\circ} \mathrm{C}$ with ambient photoperiod).

\subsection{Molecular cloning of BtAqps}

Both full-length and partial BtAqp sequences were identified by direct query searches and local BLAST searches of the University of Arizona B. tabaci sequence database (maintained by Judith Brown) and from the Short Read Archive (SRA) databases SRX022878 and SRA036954 published by Wang et al. (2010) and Xie et al. (2012), respectively. The full-length Btdripl cDNA (EU127479.1), previously referred to as BtAQP1, was obtained from an earlier study (Mathew et al. 2011). We verified variant forms of Btdrip2 present in SRAs using primers 28BtAQP3, 29BtAQP3, and 61BtAQP3 in 5'-RACE as previously described by Mathew et al. (2011). Sequences with full-length open reading frames (ORFs) corresponding to BtPrip, BtEglpA, BtEglpB1, BtEglpB2, and BtAqp12L were identified from either the University of Arizona B. tabaci sequence database or SRAs and were verified by cloning from cDNA. A partial fragment corresponding to Btbib was present in the SRAs. Whereas 3'-RACE with nested primers 31BtAQP5 and 32BtAQP5 provided the 3'-end of Btbib, several attempts using 5'-RACE failed to provide a complete cDNA 5'-end.

Full-length Btaqp coding sequences (CDS) corresponding to ORFs were confirmed by PCR amplification from B. tabaci adult cDNA using primer pairs (Table 1). All products corresponding to full-length CDS were PCR amplified using ExTaq DNA 
polymerase (Takara-Clontech, Mountain View, CA) and products were electrophoresed on a $1 \%$ agarose gel and visualized using SYBR Safe (Life Technologies, Carlsbad, CA). Bands were gel-purified using Montage DNA Gel Extraction Kit (EMD Millipore/Merck KGaA, Darmstadt, Germany) and subcloned into pCR2.1-TOPO (Life Technologies, Carlsbad, CA). Plasmid DNA was propagated in One Shot TOP10 chemically competent Escherichia coli and purified using QIAprep Spin MiniPrep Kit (Qiagen, Valencia, CA) in QIAcube robotic workstation. Inserts were sequenced with T7 and M13 Reverse vector primers by the Arizona State University DNA Core Lab (Tempe, AZ).

\subsection{Phylogenetics of hemipteran aquaporins}

The deduced B. tabaci proteins were aligned with other hemipteran peptide sequences obtained from public databases (ensembl, NCBI) using the L-INS-I algorithm of MAFFT v7.245 (Katoh and Toh, 2008). Further hemipteran sequences were obtained from opensource whole genome and transcriptome shotgun sequences (NCBI), assembled and added to the alignment as described previously (Finn et al., 2014) (Table S1). A corresponding codon alignment was generated using Pal2Nal (Suyama et al., 2006) and phylogenetically analyzed using MrBayes v3.2.2 (Ronquist and Huelsenbeck, 2003). Bayesian model parameters were nucmodel=4by4, nst.2, rates=gamma for codon alignments and aamodel=mixed for amino acid alignments. The resulting probability distributions were examined for convergence using Tracer v1.6 (tree.bio.ed.ac.uk/software/tracer/). All trees generated were processed with Archaeopteryx (Han and Zmasek, 2009), rendered with Geneious (Biomatters Ltd New Zealand) and a final tree annotated with EazyDraw (Dekorra Optics, USA). The statistical data at each node represent the posterior probabilities of the codon and amino acid alignments derived from one million MCMC generations and a burn-in of $25 \%$. To 
achieve these results, two sequences were excluded, one for the water strider (Gerris buenoi) Drip, which causes the Drip clade to co-cluster with the Bib clade, and a highly degenerate Prip2-like sequence in the glassy-winged sharpshooter (Homalodisca vitripennis).

\subsection{Bioinformatics}

The NPA motifs and ar/R selectivity sites of the BtAqps were identified through alignment with the Homo sapiens aquaporin 1 sequence (EAL24446.1). Subcellular localization predictions were made using WOLF-PSORT (Horton et al., 2007). Predictions of intra- and extracellular domains and transmembrane helices were made using TMPred (Hofmann and Stoffel, 1993), TMHMM2.0 (Krogh et al., 2001), Phobius (Käll et al., 2004), RHYTHM (Rose et al., 2009), TOPCONS (Bernsel et al., 2009), and HMMTOP (Tusnady and Simon, 2001). Isoelectric point (pI) and molecular weight (MW) were calculated using the ExPASy Molecular Biology Server of Swiss Institute of Bioinformatics. Putative glycosylation and phosphorylation sites were predicted using NetOGlyc4.0 (Steentoft et al., 2013) and NetPhos2.0 (Blom et al., 1999), respectively. Putative signal peptides were determined using Signal-BLAST (Frank and Sippl, 2008).

\subsection{RNA extraction, cDNA synthesis, and semi-quantitative reverse transcription PCR}

B. tabaci developmental stages were synchronized by placing several hundred adults inside a clip cage on a single, mature broccoli leaf and allowed to lay eggs for $48 \mathrm{~h}$. Eggs, $1^{\text {st }}$ instars, $2^{\text {nd }}-3^{\text {rd }}$ instars, $4^{\text {th }}$ instars, and adult males and females were collected and preserved at $-80{ }^{\circ} \mathrm{C}$ until RNA extraction.

For tissue dissections, B. tabaci adults were collected and held at $4{ }^{\circ} \mathrm{C}$ for $20 \mathrm{~min}$ to immobilize the whiteflies. One hundred complete gut tracts, 100 bodies without gut 
tracts, 30 head/thoraces, and 30 abdomens were dissected from female $B$. tabaci in dissection buffer [10 mM Tris-HCl, $\mathrm{pH}$ 7.4, 0.15 M NaCl, 0.01\% Triton X-100, $0.4 \mathrm{mM}$ phenylmethylsulfonyl fluoride (PMSF)] under a stereomicroscope while chilled over an ice pack.

For RNA extraction, insect and gut samples were homogenized using Kontes pestles (Fisher Scientific, Pittsburg, PA) and total RNA was extracted using TRIzol® reagent (Life Technologies). Total RNA concentration was determined using a NanoDrop ND1000 spectrophotometer (NanoDrop Technologies/Thermo Scientific, Wilmington, DE) and $1 \mu \mathrm{g}$ of total RNA was treated with DNA-free ${ }^{\mathrm{TM}}$ DNase (Ambion, Austin, TX) in a $10-\mu \mathrm{L}$ reaction to remove potential genomic DNA contamination. To prepare cDNA, $500 \mathrm{ng}$ of total RNA was used, using oligo-dT primers according to the protocol described in RETROscript Kit (Ambion). For semi-quantitative reverse transcription PCR, Btaqp-specific primers were designed to amplify an approximately 500-bp fragment of each cDNA (Table 1). As a reference gene, we designed primers amplifying a 249-bp Btrpl29 fragment (Table 1), which was empirically determined to be the most constitutive reference gene throughout development and in the tissues. The profiles were generated using Sapphire Amp Fast PCR Master Mix (Clontech Laboratories, Mountain View, CA), $12.5 \mathrm{ng}$ of each cDNA template, and $0.2 \mu \mathrm{M}$ of primers. Thermocycler conditions consisted of $95^{\circ} \mathrm{C}$ for $2 \mathrm{~min}$ followed by 30 or 35 cycles at $95{ }^{\circ} \mathrm{C}$ for $20 \mathrm{~s}, 56$ ${ }^{\circ} \mathrm{C}$ for $20 \mathrm{~s}$, and $72{ }^{\circ} \mathrm{C}$ for $30 \mathrm{~s}$, and a final extension at $72{ }^{\circ} \mathrm{C}$ for $5 \mathrm{~min}$. PCR products were separated on $2 \%$ agarose gels using a Tris/acetate/EDTA buffer system and visualized with SYBR Safe (Life Technologies).

\subsection{Expression and cellular localization in Trichoplusia ni cell culture}


To observe cellular localization, recombinant BtAqps were expressed using the pIB/V5-His TOPO TA insect cell expression vector (Life Technologies) in cultured Trichoplusia ni cells (Allele Biotechnology, San Diego, CA). Recombinant proteins were expressed as chimeras with the enhanced green fluorescent protein (EGFP) produced in frame with BtAqps at their carboxyl-termini. BtAqp-EGFP chimeras were produced by overlap extension PCR (Wurch et al., 1998). Overlap extension primers were designed such that for each of the seven tested BtAqps, the amino-terminal BtAqp (without stop codon) incorporated 15 nucleotides from the 5'-end of egfp at its 3'-end, and the carboxylterminal EGFP incorporated 15 nucleotides from the 3'-end of Btaqp (without the stop codon) at its 5'-end (Table 1).

The first PCR amplified either the full-length BtAqp cDNA including the 15 egfp nucleotides at the 3'-end or the full-length EGFP with 15 Btaqp nucleotides at the 5'-end using KOD polymerase (EMD Millipore). Thermocycler conditions consisted of 30 cycles at $98{ }^{\circ} \mathrm{C}$ for $20 \mathrm{~s}$ and $56^{\circ} \mathrm{C}$ for $30 \mathrm{~s}$. Amplimers were separated on a $1 \%$ agarose gel using a Tris/acetate/EDTA buffer system, visualized with SYBR Safe (Life Technologies), and gel purified using an EZNA gel extraction kit (Omega Bio-Tek, Norcross, GA). Purified PCR products were quantified with a NanoDrop ND1000 spectrophotometer.

To generate full-length chimeras, a second round of PCR was performed with primers containing the start codon of the Btaqp and a primer containing the egfp stop codon (Table 1). Equal amounts of each gel-purified PCR product were used as template and full-length Btaqp-egfp chimeras were generated with KOD polymerase (EMD Millipore). Thermocycler conditions consisted of 35 cycles at $95{ }^{\circ} \mathrm{C}$ for $15 \mathrm{~s}, 56^{\circ} \mathrm{C}$ for $20 \mathrm{~s}$, and 72 ${ }^{\circ} \mathrm{C}$ for $20 \mathrm{~s}$. Amplimers were separated on a 1\% agarose gel, gel purified, quantified with NanoDrop ND1000, and sub-cloned into the pIB/V5-His TOPO vector (Life 
Technologies). Plasmid DNA was propagated in One Shot TOP10 E. coli cells (Life Technologies), and then purified and sequenced as previously indicated.

With the exception that a Fluoview FV10i-LIV laser scanning confocal microscope (Olympus, Center Valley, PA) was used, transfections and fluorescence microscopy were performed according to protocols previously established (Mathew et al., 2011; Fabrick et al., 2014). T. $n i$ cells maintained as adherent cultures were transfected with $2 \mu$ g plasmid (Btaqp-egfp/pIB chimeras or egfp/pIB transfection control) using Insect Gene Juice transfection reagent (Novagen, EMD Biosciences, San Diego, CA). Forty-eight hours after transfection, the cells were washed twice with $1 \mathrm{~mL}$ IPL-41 insect media (Life Technologies) and imaged using a 60x phase contrast water-immersion objective (NA 1.2). Images were taken at $102 x$ amplification and subsequently processed in Adobe Photoshop Elements 5.0 (Adobe System Inc., San Jose, CA).

\subsection{Xenopus laevis oocyte expression and permeability assays}

Full-length Btaqp inserts were excised from pCR2.1-TOPO using restriction enzymes EcoRV and SpeI and ligated into pT7Ts oocyte expression plasmids. The Btaqp-pT7Ts constructs were linearized with $X b a \mathrm{I}$ or $S a l \mathrm{I}$, depending on the restriction sites identified in the Btaqp sequence, and cRNAs for microinjection were synthesized using T7 RNA polymerase. $X$. laevis stage $\mathrm{V}$ and VI oocytes were isolated and microinjection was performed as described before (Finn et al., 2015). Procedures relating to the care and use of $X$. laevis were approved by the Ethics Committee from IRTA in accordance with the Guiding Principles for the Care and Use of Laboratory Animals. Oocytes were transferred to modified Barth's medium (MBS) containing $88 \mathrm{mM} \mathrm{NaCl}, 1 \mathrm{mM} \mathrm{KCl,} 2.4$ $\mathrm{mM} \mathrm{NaHCO} 3,0.82 \mathrm{mM} \mathrm{MgSO}_{4}, 0.33 \mathrm{mM} \mathrm{Ca}\left(\mathrm{NO}_{3}\right)_{2} .0 .41 \mathrm{mM} \mathrm{CaCl}_{2}, 10 \mathrm{mM}$ HEPES and $25 \mu \mathrm{g} / \mathrm{mL}$ gentamycin, $\mathrm{pH} 7.5$, and injected with $50 \mathrm{~nL}$ of distilled water (negative 
control) or $50 \mathrm{~nL}$ of water solution containing $15 \mathrm{ng}$ cRNA. One day after injection, oocytes were manually defolliculated and subsequently maintained in MBS at $18{ }^{\circ} \mathrm{C}$. For determination of osmotic water permeability $\left(P_{\mathrm{f}}\right)$, the oocytes were transferred from isotonic MBS (200 mOsm) to 10-fold diluted MBS (20 mOsm) two days after injection. Oocyte swelling was followed by video microscopy using serial images at 2-s intervals during the first 20-s period using a Nikon Color View video camera coupled to a stereomicroscope (SMZ1000, Nikon). The $P_{\mathrm{f}}$ values were calculated taking into account the time-course changes in relative oocyte volume $[\mathrm{d}(\mathrm{V} / \mathrm{V} 0) / \mathrm{dt}]$, the partial molar volume of water $\left(\mathrm{V}_{\mathrm{W}}=18 \mathrm{~cm}^{3} \mathrm{~mol}^{-1}\right)$, and the oocyte surface area $(\mathrm{S})$ using the formula: $\mathrm{V}_{0}\left[\mathrm{~d}\left(\mathrm{~V} / \mathrm{V}_{0}\right) / \mathrm{dt}\right] /\left[\mathrm{SVW}\left(\mathrm{Osm}_{\text {in }}-\mathrm{Osm}_{\text {out }}\right)\right]$. The surface area of the oocyte was considered to be nine times the apparent area because of membrane folding.

Glycerol uptake by $X$. laevis oocytes expressing BtAqps was determined under isotonic conditions. Groups of 10-20 oocytes injected with water or $15 \mathrm{ng}$ aquaporin cRNA were incubated at room temperature in $200 \mu \mathrm{l}$ of isotonic MBS containing $5 \mu \mathrm{M}$ $(20 \mu \mathrm{Ci})$ of $\left[1,2,3-{ }^{3} \mathrm{H}\right]$-glycerol $\left(50 \mathrm{Ci} \mathrm{mmol}^{-1}\right)$ (American Radiolabelled Chemicals Inc., St. Louis, MO) and cold glycerol at $1 \mathrm{mM}$ final concentration. After 10-min exposure to radioactive compounds (including zero time for subtraction of the signal from externally bound solute), oocytes were washed rapidly in ice-cold MBS three times, and individual oocytes were dissolved for $1 \mathrm{~h}$ in $400 \mathrm{ml}$ of $10 \%$ SDS before scintillation counting. Statistical analyses were performed using GraphPad Prism 6 (GraphPad Software, Inc., La Jolla, CA) and functionality of aquaporins was compared with the water-injected control using Student's $t$-test with significance level $\alpha=0.05$.

\section{Results}

\subsection{Cloning, topology, and bioinformatics}


NextGen transcriptome sequencing of $B$. tabaci has provided unprecedented resources to mine for previously uncharacterized gene transcripts. Here, we identified a total of ten sequences corresponding to eight aquaporin paralogs from three $B$. tabaci transcriptomes, including two publically available SRAs (SRX018661 and SRA036954) and a third $B$. tabaci midgut and salivary gland database not currently publically available (maintained by Judith Brown, University of Arizona). Seven full-length aquaporin cDNAs were PCR amplified, cloned, and sequence verified (EU127479 and KT963825KT963832). In addition, a partial cDNA sequence for a putative Btbib was cloned; however, repeated attempts to obtain the missing 5'-end failed.

B. tabaci dripl previously characterized as BtAQP1 (EU127479.1) by Mathew et al. (2011) encodes a 262 amino acid ORF with a predicted isoelectric point (pI) and molecular weight (MW) of 6.57 and $27.3 \mathrm{kDa}$, respectively (Table 2). BtDrip1 has two NPA motifs and Phe71, His198, Ala207, and Arg213 that comprise the ar/R selectivity filter (Fig. 1). Furthermore, a putative mercury-sensitive Cys is located two residues upstream of the first NPA motif. Topology predicting tools calculated that BtDrip1 has six TM regions with intracellular amino- and carboxyl-termini (Table 3). BtDrip1 has seven putative $\mathrm{O}$-glycosylation sites and 15 putative phosphorylation sites, but no Nglycosylation sites or signal peptide (Table S2).

We identified three amino-terminal isoforms of BtDrip2 (variant 1, 2, and 3) by 5'RACE. Each Btdrip2 CDS (924 bp for Btdrip2_v1; 771 bp for Btdrip2_v2, and 567 bp for Btdrip2_v3) encodes 307, 256, and 188 amino acids, with pIs and MWs of 8.89/32.7 kDa, 8.07/26.8 kDa, and 6.93/19.4 kDa, respectively (Table 2). All three Btdrip2 RNA variants contain the 567-bp CDS that comprise Btdrip2_v3 and RT-PCR cloning verified that all three transcripts are present in B. tabaci. While BtDrip2_v1 and BtDrip2_v2 are predicted to possess the required structures to form a functional aquaporin (NPA motifs 
and ar/R selectivity site), the ORF of BtDrip2_v3 is truncated at its amino-terminus compared to the two other variants. BtDrip2_v1 and BtDrip2_v2 have ar/R selectivity sites Phe113/62, His240/189, Ala249/198, and Arg255/204. Translation of Btdrip2_v3 RNA results in a protein completely lacking an amino-terminal domain as well as much of the first TM domain, including the Phe residue that contributes to the ar/R filter (Fig. 1). Topology predictions indicate that BtDrip2_v1 and BtDrip2_v2 have six TM regions, with intracellular amino- and carboxyl-termini. BtDrip2_v3 is predicted to have four or five TM regions with an extracellular amino-terminus and an intracellular carboxylterminus (Table 3). BtDrip2 is predicted to have three putative O-glycosylation sites and 14 phosphorylation sites, but no N-glycosylation sites or signal peptide (Table S2).

B. tabaci prip consists of a 792 bp CDS encoding 263 amino acids, with predicted $\mathrm{pI}$ and MW of 5.92 and $27.4 \mathrm{kDa}$, respectively (Table 2). BtPrip has two conserved NPA motifs and Phe62, His186, Thr195, and Arg201 comprise the ar/R selectivity site. TM topology analysis predicts the presence of six TM regions for BtPrip with intracellular amino- and carboxyl-termini (Table 3). BtPrip contains one putative O-glycosylation site and seven putative phosphorylation sites, but no $\mathrm{N}$-glycosylation sites or signal peptide (Table S2).

B. tabaci also contains an extended repertoire of Eglps, belonging to two clades, A and B. The 789-bp CDS of B. tabaci eglpA encodes 262 amino acids, with predicted pI and MW of 7.3 and $28.3 \mathrm{kDa}$, respectively (Table 2). BtEglpA has two conserved NPA motifs and Phe28, Ser160, Ala169, and Arg175 comprise the ar/R selectivity site (Fig. 1). Based on previous results (Finn et al., 2015), the substitution of a Ser residue for His within the ar/R selectivity filter likely plays an important role for differentiation of permeable solutes through the central pore of BtEglpA. TM topology analysis for BtEglpA predicts five to six TM-spanning regions (Table 3). BtEglpA also contains a 
putative O-glycosylation site and 13 putative phosphorylation sites (Table S2). No Nglycosylation sites or signal peptides are predicted. We identified two unique full-length BtEglpBs by RT-PCR and cloning, with BteglpB1 having a CDS of 840 bp encoding 279 amino acids and BteglpB2 having a CDS of 777 bp encoding 258 amino acids. The predicted pI and MW of BtEglp1 are 8.47 and $30.3 \mathrm{kDa}$, respectively, and 8.56 and 28.5 kDa for BtEglp2 (Table 2). Both BtEglpB1 and BtEglpB2 possess two NPA motifs and have conserved Phe/Leu/Ser/Arg ar/R selectivity residues (Phe68/Leu196/Ser205/Arg211 for BtEglpB1 and Phe63/Leu190/Ser199/Arg205 for BtEglpB2), in which the classical aquaporin His residue is substituted by Leu (Finn et al., 2015) (Fig. 1). Topology analysis predicts that both BtEglpB1 and BtEglpB2 contain six TM domains with intracellular amino- and carboxyl-terminal domains (Table 3). Whereas BtEglpB1 contains two putative $\mathrm{O}$-glycosylation sites and 11 putative phosphorylation sites, BtEglpB2 is predicted to contain two O-glycosylation sites and 16 phosphorylation sites (Table S2). No N-glycosylation sites or signal peptides are predicted for either BtEglpB1 or BtEglpB2.

The unorthodox Btaqp12L consists of a 789 bp CDS encoding 262 amino acids, with putative pI and MW of 6.98 and $29.0 \mathrm{kDa}$, respectively (Table 2). The NPA motifs and ar/R residues of BtAqp12L are not conserved, with the first and second motifs consisting of 93-CPY-95 and 210-NPV-212, respectively, and the ar/R constriction site consisting of Tyr72, Val198, Gly207, Leu213 (Fig. 1). TM topology predictions for BtAqp12L varied, with five (RHYTHM, HHMTOP), six (TMPred), or seven (TMHMM, Phobius, TOPCONS) TM-spanning regions (Table 3). BtAqp12L contains two putative Oglycosylation sites and five phosphorylation sites, but no $\mathrm{N}$-glycosylation sites or signal peptide (Table S2). 


\subsection{Phylogeny}

Arthropod aquaporins are classified into three major grades comprising six major subfamilies that include the big brain cation channel proteins (Bib), the Drosophila integral protein (Drip)-like aquaporins, the Pyrocoelia rufa integral protein (Prip)-like aquaporins, the recently described entomoglyceroporins (Eglps), the distantly related aquaporin 12-like (Aqp12L) subfamily, and in older hexapod lineages - the classical aquaglyceroporins (Glps) (Finn et al., 2015; Stavang et al., 2015). Sequence alignment and phylogenetic analysis of BtAqp sequences with 104 aquaporins from 11 superfamilies of hemipteran insects revealed that the hemipteran aquaporin superfamily consists of two grades of channel, the classical aquaporin 4-related channels (Bib, Drip, Prip and Eglp), and the unorthodox Aqp12L channels (Fig. 2). These data indicate that, as in holometabolous insects, hemipterans have lost the classical Glps found in the older hexapod lineages. The overall tree topology shows separation of the hemipteran aquaporin superfamily into seven major clusters, with three subclusters representing duplicated members of the Eglp subfamily (EglpA, -B and -C). Amongst these clusters, B. tabaci has single copies of the Bib, Prip, EglpA and Aqp12L subfamilies, but duplicated members of the Drip (BtDrip1 and BtDrip2) and EglpB (EglpB1 and -B2) subfamilies. The molecular repertoire of $B$. tabaci aquaporin paralogs is further expanded through the existence of three isoforms of Drip2 (BtDrip2_v1, BtDrip2_v2, and BtDrip2_v3) in which the amino-terminal domain is alternatively spliced.

\subsection{Temporal expression and tissue localization}

Transcriptional expression profiles of the Btaqps were examined using end-point RTPCR (30 and 35 cycles) over the life cycle of B. tabaci $\left(1^{\text {st }}\right.$ instars, combined $2^{\text {nd }}-3^{\text {rd }}$ instars, $4^{\text {th }}$ instars, and adults) (Fig. 3A) and in several different tissues (Fig. 3B). Btdripl 
transcripts are present in all life stages, but are less abundant in eggs and adults than in the nymphal stages (Fig. 3A). In adults, Btdripl RNA is most abundant in the gut, but is present within other tissues, including body minus gut, head/thoraces, and abdomens. Using RT-PCR and an amplicon encompassing all three Btdrip2 variant transcripts, we found transcripts primarily in nymphs, but some transcripts could be detected in eggs and within in the adult body minus gut. Btdripl transcripts are present in all life stages, but are more abundant in nymphs than in adults. In adult tissues, Btprip transcription is highest in body minus gut and/or abdomen. Transcription of BteglpB1 and BteglpB2 appears to be fairly constitutive, with transcripts present in all life stages and most tissues we tested. Both BteglpB transcripts are less abundant in adults than immature stages and BteglpB2 is minimal in head-thoraces. The unorthodox Btaqp12L transcripts are most abundant in immature whiteflies, but are also found specifically within the guts of adults.

\subsection{Cellular localization}

We used WOLF-PSORT (Horton et al., 2007) for subcellular localization prediction of each translated BtAqp sequence. All seven BtAqps (including the three BtDrip2 splice variants) are predicted to localize within the plasma cell membrane. To examine cellular localization, chimeric recombinant BtAqp proteins tagged at their carboxyl-termini with EGFP were produced in cultured $T$. $n i$ cells and observed by confocal fluorescence microscopy. We observed several patterns of localization for the recombinant BtAqpEGFPs, including those that primarily translocate to the plasma membrane, those that show some localization at cell surface but have significant retention within the cellular localization machinery (e.g. Golgi and endoplasmic reticulum), and those that are primarily intracellular. 
BtDrip1-EGFP localized to the plasma cell membrane (Fig. 4B), which corroborated our earlier observations (Mathew et al., 2011; Fabrick et al., 2014). BtDrip2_v1-EGFP, BtDrip2_v2-EGFP, BtPrip-EGFP, BtEglpB1-EGFP, and BtEglpB2-EGFP showed some localization to the plasma membrane, but significant fluorescence was also observed within the cell, indicating some of the proteins remained within the secretory pathway (Fig. 4C, 4D, 4F, 4H, 4I). BtDrip2_v3-EGFP, which is the most truncated BtDrip2 isoform at its amino-terminus, localized within the cytoplasm of the transfected T. ni cells (Fig. 4E). BtEglpA-EGFP and BtAqp12L-EGFP were also retained with the cells (Fig. 4G, 4J). These results suggest that the cellular trafficking and/or protein folding machinery differ between $B$. tabaci and $T$. $n i$ cells or that these proteins may have an intracellular function. This is of particular interest for BtAqp12L, which shares similarity with other unorthodox aquaporins that have low sequence similarity to classical aquaporins, have altered NPA motifs, and localize intracellularly in vivo where they may function in the transport of intracellular water through organelles (Finn and Cerdà, 2015; Fabrick et al., 2014; Ishibashi, 2006).

\subsection{Oocyte permeability assays}

The water and glycerol transport functions of BtAqps were tested using the X. laevis oocyte heterologous expression system. In these experiments, aquaporin cRNAs were expressed in oocytes and the $P_{\mathrm{f}}$ determined by measuring the rates of swelling under hypotonic conditions (Fig. 5A). We observed significant $(p<0.05)$ water permeability for oocytes injected with cRNA corresponding to human aquaporin 1 (hAQP1) and aquaporin 3 (hAQP3) (positive controls) as well as for BtDrip1, BtDrip2_v1, BtPrip, BtEglpA, BtEglpB1, and BtEglpB2. Amongst the BtAqps, the mean $P_{\mathrm{f}}$ was highest in oocytes expressing BtDrip1 $\left(934.3 \pm 33.1 \mu \mathrm{m} \mathrm{s}^{-1}\right)$, which was comparable with that of 
oocytes expressing the human aquaporins $\left(1,096.0 \pm 70.9 \mu \mathrm{m} \mathrm{s}^{-1}\right.$ for hAQP1; $812.2 \pm$ $47.3 \mu \mathrm{m} \mathrm{s}^{-1}$ for hAQP3). Although less than other aquaporins, water permeability was detected for BtEglpA $\left(91.9 \pm 15.8 \mu \mathrm{m} \mathrm{s}^{-1}\right)$, BtEglpB1 $\left(294.2 \pm 27.3 \mu \mathrm{m} \mathrm{s}^{-1}\right)$ and BtEglpB2 $\left(588.5 \pm 35.4 \mu \mathrm{m} \mathrm{s}^{-1}\right)$, indicating that these putative Eglps can transport water. No significant water permeability with respect to the water-injected oocytes was observed for BtDrip2_v2, BtDrip2_v3, or BtAqp12L.

For glycerol uptake in $X$. laevis oocytes expressing BtAqps, significant $(p<0.05)$ uptake was observed in oocytes expressing BtDrip1 $\left(38.8 \pm 2.8\right.$ pmol oocyte $\left.^{-1}\right)$, BtEglpA $\left(88.8 \pm 7.8\right.$ pmol oocyte $\left.{ }^{-1}\right)$, BtEglpB1 $\left(167.6 \pm 3.7\right.$ pmol oocyte $\left.{ }^{-1}\right)$, BtEglpB2 $(126.1 \pm 7$. 9 pmol oocyte $\left.^{-1}\right)$, and in the hAQP3 positive control $\left(148.8 \pm 5.8\right.$ pmol oocyte $\left.{ }^{-1}\right)$. Glycerol uptake in BtDrip2 (all 3 variants), BtPrip, and BtAqp12L was not significantly different from that observed in the control and hAQP1 oocytes. BtEglpA, BtEglpB1, and BtEglpB2 oocytes showed a significant $(p<0.05)$ accumulation of ${ }^{3} \mathrm{H}$-glycerol (Fig. 5B) and water permeability (Fig. 5A), indicating that these proteins are likely efficient transporters of both glycerol and water.

\section{Discussion}

Aquaporins are indispensable water and solute channels needed to maintain homeostasis in living organisms. In insects, aquaporins relieve pressure imposed by osmotically imbalanced diets, remove excess fluids and excretions, maintain fluid homeostasis during oogenesis, stabilize membranes and enzymes for anhydrobiosis and cryoprotection, are involved in water movement in respiration, and prevent desiccation during molting and egg hatching (Campbell et al., 2008; Drake et al., 2010, Duchesne et al., 2003, Fabrick et al., 2014; Goto et al., 2011, 2015; Herraiz et al., 2011; Kikawada et al., 2008; Le Cahérec et al., 1997; Liu et al., 2011; Martini et al., 2004; Maruyama et al., 
2015; Mathew et al., 2011; Nagae et al., 2013; Philip et al., 2011; Spring et al., 2009; Staniscuaski et al., 2013; Yi et al., 2011). Because of their major importance in these physiological processes, aquaporins may serve as potential targets for insect pest management, especially in phloem-feeding hemipterans where imbibition of large volumes of liquid diet is required to meet nutrient requirements (Douglas, 2006; Ibanez et al., 2014; Mathew et al., 2011; Shakesby et al., 2009; Tzin et al., 2015; Wallace et al., 2012). B. tabaci is of particular interest because of its stature as a pest to food, fiber, and ornamental crops. Preliminary studies of BtAqps indicated that it contains at least seven aquaporin paralogs of which at least three were classified as classical aquaporins (two Drips and one Prip) (Jing et al., 2016; Mathew et al., 2011). Recently, Raza et al. (2016) showed that gene silencing of Btdripl by oral RNA interference (RNAi) causes reduced survival of $B$. tabaci adults, indicating that this protein may be important in maintaining homeostasis. Here, we expand previous results and show that B. tabaci MEAM1 possesses eight unique BtAqp paralogs, of which we characterize seven using phylogenetics, expression profiling, cellular localization, and functional analysis.

Phylogenetic comparisons of hemipteran aquaporins show that the classical aquaporin family is comprised of several major subfamilies, including the Drips, Prips, Bibs, and the recently described entomoglyceroporins (Eglps), which we show here consist of three previously undescribed clades, EglpA, EglpB, and EglpC. As in other arthropods (Finn and Cerdà, 2015; Stavang et al. 2015) hemipterans also have members that belong to the distantly related Aqp12L family. Although the classical Glps are found in older hexapod lineages, as well as members of the psocodean order, hemipterans do not appear to have this family of aquaporins, which is consistent with the reported loss of these channels in the common ancestor of the Condylognatha (Finn et al., 2015). 
For B. tabaci, seven of the eight identified BtAqps belong to the classical grade of aquaporins. These include two Drips (BtDrip1 and BtDrip2), BtPrip, BtBib (of which we were only able to obtain a partial cDNA), and three Eglps (BtEglpA, BtEglpB1, and BtEglpB2). The eighth BtAqp, BtAqp12L, belongs to a separate family of unorthodox aquaporins, which share similarity with the vertebrate aquaporin 12-like proteins.

The Drip subfamily is the most studied group of insect aquaporins. In B. tabaci, we previously characterized BtDrip1 (earlier referred to as BtAQP1), showing that this aquaporin is a very effective water-selective channel primarily expressed in the filter chamber and hindgut of $B$. tabaci and likely plays an important role in shunting excess dietary water from the filter chamber to the ileum of the hindgut (Mathew et al., 2011). BtDrip1 has two NPA motifs, conserved ar/R constriction residues, and a putative conserved mercury-sensitive Cys residue (Fig. 1). Although previously undetected in the embryonic stage, modification of the transcriptional profiling method (increased number of amplification cycles) confirmed constitutive expression of BtDrip1 throughout all stages (Mathew et al., 2011). B. tabaci dripl transcripts (Fig. 3) and BtDrip1 protein (Mathew et al., 2011) indicate that this aquaporin is most abundant in gut tissue and specifically localizes within the filter chamber and anterior hindgut. BtDrip1 is found primarily in the plasma membrane of cells (Fig. 4B) and is an efficient transporter of water (Fig. 5A; Mathew et al., 2011), but not glycerol (Fig. 5B; Mathew et al., 2011). A small yet significant uptake of glycerol was observed for BtDrip1 (Fig. 5B), however the physiological relevance is unknown due to the extreme sensitivity of the radiolabel assay used in this study.

In contrast to other hemipterans studied to date, B. tabaci has a second Drip, named BtDrip2. We identified three isoforms of BtDrip2 in B. tabaci, each of which has a unique 5 ' start site. Our phylogenetic data reveal that both amino-terminal and carboxyl- 
terminal aquaporin splice-variants are prevalent amongst the Hemiptera. For example, amino-terminal splice variants are observed in the Drip, EglpA, and EglpC-type channels, while carboxyl-terminal splice variants occur in the Prip and EglpC-type channels. Similarly, splice variants have been reported for human kidney AQP2 (Ma et al., 1996), human brain mitochondria AQP9 (Amiry-Moghaddam et al., 2005), rat AQP4 (Moe et al., 2008), Anopheles gambiae aquaporin 1 (Tsujimoto et al., 2013), Lygus hesperus EglpA (previously LhAQP2, Fabrick et al., 2014), and Belgica antarctica Prip (Goto et al., 2011) and it was reported that some of these variants differ in localization or function. For example, in A. gambiae, the aquaporin 1 variant A is specifically expressed in female ovaries, while variant B is expressed in the midgut, hindgut, Malpighian tubules and the head (Tsujimoto et al., 2013). Rat AQP4 has 6 isoforms (a-f) and some are localized intracellularly and do not transport water when expressed in X. laevis oocytes (Moe et al., 2008). Here, the two longest BtDrip2 variants (v1 and v2) contain six TM regions, two NPA motifs, conserved water-specific ar/R selectivity residues, putative mercury sensitive Cys residues (Fig. 1), and are translocated to the plasma cell membrane (Fig. 4C and 4D). In contrast, BtDrip2_v3 is truncated at its amino-terminus, lacks the first TM domain and Phe residue of the ar/R selectivity filter (Fig. 1), does not translocate to the cell surface (Fig. 4E). X. laevis oocyte functional assays show that only the longest variant (BtDrip2_v1) forms a functional water channel (Fig 5A), indicating that shorter variants may not produce functional proteins. Transcriptional profiling of BtDrip2 further shows that it is present primarily in immature life stages, but not adult tissues (Fig. 3). Our results agree with the in silico finding of Jing et al. (2016), claiming that BtDrip2 likely does not play a significant osmoregulatory role in the adult whitefly, but rather may be important for maintaining water homeostasis during the hatch and molting process. 
B. tabaci has one Prip, corresponding to aquaporin 3 in Jing et al. (2016). This BtPrip has two predicted NPA motifs and conserved water-selective ar/R constriction residues (Fig. 1). When expressed in T. ni cells, BtPrip localized to the plasma cell membrane but was also intracellular (likely within the secretory pathway) (Fig. 4F), whereas oocyte assays confirmed water-selectivity, but no glycerol transport (Fig. 5). BtPrip transcripts are most abundant in immature stages (Fig. 3A), but are found in low abundance in the abdomen and gut of adult whiteflies. In other insects, Prips have a variety of physiological functions. For example, Blattella germanica Prip is present in the adult ovary, fat body, and muscles, and plays a role in fluid homeostasis during oogenesis (Herraiz et al., 2011). The Bombyx mori Prip is found in the oocyte plasma membrane within ovaries and functions during oocyte growth (Maruyama et al., 2015). Other Prips are involved in hydration and dehydration in response to environmental stress (Kikawada et al., 2008). Our results indicate that BtPrip may have similar functional roles of water transport within ovaries, fat bodies, or muscles of $B$. tabaci.

Recent findings of Finn et al. (2015) demonstrate that several modern lineages of hexapods, including the highly diverse holometabolan insects lack Glps for the transport of glycerol, but rather contain Eglps that evolved from water-selective classical aquaporins via mutations within the ar/R selectivity filter His residue. The previously described Glps in B. mori, Grapholita molesta, Acyrthosiphon pisum, Rhodnius prolixus, and Aedes aegypti actually phylogenetically resolve within the Eglp clades (Drake et al., 2015; Kataoka et al., 2009a/b; Staniscuaski et al., 2013; Wallace et al., 2012). Here, we likewise show that the hemipteran $B$. tabaci has three Eglps belonging two unique clades (BtEglpA and BtEglpB), all containing an altered ar/R selectivity filter (Fig. 1). The ar/R His residue is replaced by Ser in BtEglpA and by Leu for both BtEglpBs (BtEglpB1 and 
BtEglpB2). These mutations are consistent with the presently observed ability of the channels to transport glycerol.

As in other major lineages of arthropods, B. tabaci possesses a phylogenetically unique Aqp12-like member of the unorthodox grade of channels (Stavang et al., 2015). BtAqp12L has non-canonical NPA motifs (CPY and NPV) and non-conserved ar/R selectivity residues (Phe, His, Gly, Arg are replaced with Tyr, Val, Gly, Leu, respectively) (Fig. 1). In addition to having poor sequence conservation with other aquaporins and atypical NPA motifs, Aqp12Ls localize as sub-cellular aquaporins and are thought to participate in the transport of intracellular solutes through organelles (Ishibashi, 2006; Fabrick et al., 2014). Likewise, when we expressed BtAqp12L in T. ni cells, it exhibited diffuse cytosolic distribution (Fig. 4J). Furthermore, BtAqp12L has a putative ER-localization signal (residues 258-KSKL-261) near its carboxyl-terminus (Cosson and Letourneur, 1994; Fabrick et al., 2014).

In conclusion, we describe eight $B$. tabaci aquaporins using proposed phylogeny for arthropods by Finn et al. (2015) and Stavang et al. (2015). We show that B. tabaci has seven classical aquaporins belonging to the Bib, Drip, Prip, and Eglp subfamilies, and one unorthodox aquaporin (Aqp12L). Our data further show that hemipteran insects duplicated the Eglp channels early in their evolution to generate at least three separate clades (EglpA, -B and -C), and express several amino-terminal or carboxyl-terminal isoforms of the Drip, Prip, EglpA and EglpC-type channels. Of these subfamilies, the Eglps represent an important advance in arthropod evolution, where $B$. tabaci and other hemipterans possess glycerol-transporting channels that evolved independently of the classical Glps from other water-selective aquaporin predecessors. Each BtAqp may play a unique role in the maintenance of water balance and/or other unknown cryptic functions. Gene knockdown/knockout is needed to further discern the in vivo function of each 
BtAqp, which represents an important prerequisite for the practical development of novel biopesticides targeting pest aquaporins.

Table 1. Nucleotide primers used to obtain full-length Bemisia tabaci aquaporins, rapid amplification of cDNA ends (5'- and 3 '-RACE), reverse transcriptase-PCR (RT-PCR), and for sub-cloning into pIB expression vector.

\begin{tabular}{|c|c|c|c|c|}
\hline Primer & Primer DNA sequence & Direction & Gene Name & Application \\
\hline 16AQUA5 & ATGGAGGACATATCATCTTCCGGCGAAG & sense & Btdripl & cloning CDS \\
\hline 15AQUA3 & GAAATCATAAGAGCTCTCATCCGATCT & antisense & Btdripl & cloning CDS \\
\hline 74BtAQP5 & ATGAAACAGTCGCATCCTCTC & sense & Btdrip2_v1 & cloning CDS \\
\hline 72BtAQP5 & ATGAAAATGAGTGCAGTGATATCG & sense & Btdrip2_v2 & cloning CDS \\
\hline 45BtAQP5 & ATGGTCCAGTCAATTGGTCA & sense & Btdrip2_v3 & cloning CDS \\
\hline 73BtAQP3 & TCAACTTGATTGGAAGTCATAGGA & antisense & Btdrip2_v1-3 & cloning CDS \\
\hline 51BtAQP5 & ATGGCAGCCAGTCTGAAGAAC & sense & Btprip & cloning CDS \\
\hline 52BtAQP3 & TTAGTTATTTGCAGTTTCAACAGATTT & antisense & Btprip & cloning CDS \\
\hline 53BtAQP5 & ATGCTCATGTTTTTTGGCTGCAT & sense & BteglpA & cloning CDS \\
\hline 54BtAQP3 & TTATGAGTTGTTTGCTTTTCTTTTGG & antisense & BteglpA & cloning CDS \\
\hline 79BtAQP5 & ATGCCGTTCCAGAAAGATCC & sense & BteglpB1 & cloning CDS \\
\hline 42BtAQP3 & TTACACTTCATCGCCGGAAT & antisense & BteglpB1 & cloning CDS \\
\hline 49BtAQP5 & ATGTCCATTCGTGGAGCGTTTAC & sense & BteglpB2 & cloning CDS \\
\hline 50BtAQP3 & TTATATGTGTGAATTTGGCCGAATTTCT & antisense & BtaglpB2 & cloning CDS \\
\hline 47BtAQP5 & ATGGGGACCGCCAGTGTGT & sense & Btaqp12L & cloning CDS \\
\hline 48BtAQP3 & TCAGTGCAATTTTGATTTTTGAGTG & antisense & Btaqp12L & cloning CDS \\
\hline 31BtAQP5 & GGGAGAGTCCTCCACGACCA & sense & Btbib & 3'-RACE \\
\hline 32BtAQP5 & CGGCTCGGAATGGAGAATGT & sense & Btbib & 3'-RACE \\
\hline 28BtAQP3 & GAGATCGCCAAGCCACAGGT & antisense & Btdrip2 & 5'-RACE \\
\hline 29BtAQP3 & ATGGGGCCAGCCCAATAAAC & antisense & Btdrip2 & 5'-RACE \\
\hline 61BtAQP3 & CGATGATGAGACCGAATGTG & antisense & Btdrip2 & 5'-RACE \\
\hline 80BtAQP5 & AGTGCCCGATATTCGAGATG & sense & Btdripl & RT-PCR \\
\hline 81BtAQP3 & GCCCAGTACACCCAATGTTT & antisense & Btdripl & RT-PCR \\
\hline 60BtAQP5 & CACATTCGGTCTCATCATCG & sense & Btdrip2 & RT-PCR \\
\hline 73BtAQP3 & TCAACTTGATTGGAAGTCATAGGA & antisense & Btdrip2 & RT-PCR \\
\hline 82BtAQP5 & TTGGGAGTCCAGGAAGTGAC & sense & Btprip & RT-PCR \\
\hline
\end{tabular}




\begin{tabular}{|c|c|c|c|c|}
\hline 83BtAQP3 & GACAGCAGCTCCGAAAGAAC & antisense & Btprip & RT-PCR \\
\hline 84BtAQP5 & TCCAGAAAGATCCACCCAAC & sense & BteglpB1 & RT-PCR \\
\hline 85BtAQP3 & CGAAACTCCTTGCTGGATTC & antisense & BteglpB1 & RT-PCR \\
\hline 86BtAQP5 & ATTCGTGGAGCGTTTACACC & sense & BteglpB2 & RT-PCR \\
\hline 87BtAQP3 & TTGCTGGGTTCATACTGCTG & antisense & BteglpB2 & RT-PCR \\
\hline 88BtAQP5 & GGCTTGTCTCAGGGTTTGC & sense & BteglpA & RT-PCR \\
\hline 89BtAQP3 & AGCGACCCAGTAAACCCAAT & antisense & BteglpA & RT-PCR \\
\hline 90BtAQP5 & CTCGCAGTGTCCACCAGTTA & sense & Btaqp12L & RT-PCR \\
\hline 91BtAQP3 & ATCCCCCGGAGTAATTGAAG & antisense & Btaqp12L & RT-PCR \\
\hline 1BtRPL295 & GCTTCCCGTGTGAGGTTTTG & sense & Btrpl29 & RT-PCR \\
\hline 2BtRPL293 & ACGGGACTTATTCTGGCGTA & antisense & Btrpl29 & RT-PCR \\
\hline AQP1_EGFP & AGCTCTTATGATTTCATGGTGAGCAAGGGC & sense & Btdripl & pIB \\
\hline EGFP_AQP1 & GCCCTTGCTCACCATGAAATCATAAGAGCT & antisense & Btdripl & pIB \\
\hline AQP2A_EGFP & GACTTCCAATCAAGTATGGTGAGCAAGGGC & sense & Btdrip2_v1 & $\mathrm{pIB}$ \\
\hline EGFP_AQP2A & GCCCTTGCTCACCATACTTGATTGGAAGTC & antisense & Btdrip2_v1 & pIB \\
\hline AQP2B_EGFP & GACTTCCAATCAAGTATGGTGAGCAAGGGC & sense & Btdrip2_v2 & pIB \\
\hline EGFP_AQP2B & GCCCTTGCTCACCATACTTGATTGGAAGTC & antisense & Btdrip2_v2 & pIB \\
\hline AQP2C_EGFP & GACTTCCAATCAAGTATGGTGAGCAAGGGC & sense & Btdrip2_v3 & pIB \\
\hline EGFP_AQP2C & GCCCTTGCTCACCATACTTGATTGGAAGTC & antisense & Btdrip2_v3 & pIB \\
\hline AQP3_EGFP & GAAACTGCAAATAACATGGTGAGCAAGGGC & sense & Btprip & pIB \\
\hline EGFP_AQP3 & GCCCTTGCTCACCATGTTATTTGCAGTTTC & antisense & Btprip & pIB \\
\hline AQP4_EGFP & TCCGGCGATGAAGTGATGGTGAGCAAGGGC & sense & BteglpB1 & pIB \\
\hline EGFP_AQP4 & GCCCTTGCTCACCATCACTTCATCGCCGGA & antisense & BteglpB1 & pIB \\
\hline AQP5_EGFP & CCAAATTCACACATAATGGTGAGCAAGGGC & sense & BteglpB2 & pIB \\
\hline EGFP_AQP5 & GCCCTTGCTCACCATTATGTGTGAATTTGG & antisense & BteglpB2 & pIB \\
\hline AQP6_EGFP & AAAGCAAACAACTCAATGGTGAGCAAGGGC & sense & BteglpA & pIB \\
\hline EGFP_AQP6 & GCCCTTGCTCACCATTGAGTTGTTTGCTTT & antisense & BteglpA & pIB \\
\hline AQP7_EGFP & AAATCAAAATTGCACATGGTGAGCAAGGGC & sense & Btaqp12L & $\mathrm{pIB}$ \\
\hline EGFP_AQP7 & GCCCTTGCTCACCATGTGCAATTTTGATTT & antisense & Btaqp12L & pIB \\
\hline EGFP & TTACTTGTACAGCTCGTCCAT & antisense & $e g f p$ & pIB \\
\hline
\end{tabular}


Table 2. Full-length aquaporin cDNAs from Bemisia tabaci.

\begin{tabular}{cccccc}
\hline BtAqp & $\begin{array}{c}\text { NCBI accession } \\
\text { number }\end{array}$ & CDS (bp) & ORF (aa) $^{\mathrm{b}}$ & pI & MW (dalton) \\
\hline BtDrip1 & EU127479 & 789 & 262 & 6.57 & 27,282 \\
BtDrip2_v1 & KT963825 & 924 & 307 & 8.89 & 32,739 \\
BtDrip2_v2 & KT963826 & 771 & 256 & 8.07 & 26,843 \\
BtDrip2_v3 & KT963827 & 567 & 188 & 6.93 & 19,447 \\
BtPrip & KT963828 & 792 & 263 & 5.92 & 27,393 \\
BtEglpA & KT963831 & 789 & 262 & 7.03 & 28,273 \\
BtEglpB1 & KT963829 & 840 & 279 & 8.47 & 30,273 \\
BtEglpB2 & KT963830 & 777 & 258 & 8.56 & 28,504 \\
BtAqp12L & KT963832 & 789 & 262 & 6.98 & 29,008 \\
\hline
\end{tabular}

${ }^{\mathrm{a}}$ Total number of nucleotides in BtAqp sequence obtained from PCR cloning.

${ }^{\mathrm{b}}$ Total number of amino acids in BtAqp sequence obtained by translation of CDS.

Table 3. Prediction of intra/extracellular and transmembrane topology for Bemisia tabaci aquaporins.

\begin{tabular}{cccccccc}
\hline & \multicolumn{2}{c}{$\begin{array}{c}\text { Number of predicted transmembrane } \\
\text { helices by topology prediction tools }\end{array}$} & & & Consensus position \\
\cline { 2 - 3 } \cline { 6 - 7 } & TMPred & $\begin{array}{c}\text { TMHMM } \\
\text { Phobius } \\
\text { TOPCONS }\end{array}$ & $\begin{array}{c}\text { RHYTHM } \\
\text { HMMTOP }\end{array}$ & & $\begin{array}{c}\text { Amino- } \\
\text { terminus }\end{array}$ & $\begin{array}{c}\text { Carboxyl- } \\
\text { terminus }\end{array}$ \\
BtDrip1 & 6 & 6 & 6 & & Inside & Inside \\
BtDrip2_v1 & 7 & 6 & 6 & & Inside & Inside \\
BtDrip2_v2 & 6 & 6 & 6 & & Inside & Inside \\
BtDrip2_v3 & 4 & 4 & 5 & & Outside & Inside \\
BtPrip & 6 & 6 & 6 & & Inside & Inside \\
BtEglpA & 6 & 5 & 5 & & Outside & Inside \\
BtEglpB1 & 6 & 6 & 6 & & Inside & Inside \\
BtEglpB2 & 6 & 6 & 6 & & Inside & Inside \\
BtAqp12L & 6 & 7 & 5 & & Outside & Inside \\
\hline
\end{tabular}

\footnotetext{
${ }^{\text {a }}$ Predictions of intra/extracellular domains and transmembrane helices were made using TMPred (Hofmann and Stoffel, 1993), TMHMM2.0 (Krogh et al., 2001), Phobius (Käll et al., 2004), RHYTHM (Rose et al., 2009), TOPCONS (Bernsel et al., 2009), and HMMTOP (Tusnady and Simon, 2001).

${ }^{\mathrm{b}}$ The amino- and carboxyl-terminal ends of BtAqps were predicted to be either intracellular or extracellular based on consensus positions from the six different prediction software programs.
} 


\section{Figure legends}

Figure 1. Amino acid sequence alignment of the Bemisia tabaci aquaporins. The deduced amino acid sequences of the BtAqps were aligned using CLUSTALW. NPA motifs are highlighted in grey. Residues that correspond to the ar/R selectivity site from Homo sapiens AQP1 (F56, H180, C189, R195; EAL24446.1) are highlighted in pink, orange, red, and yellow, respectively. Underlined and highlighted in orange are the ar/R selectivity residues important for glycerol transport in entomoglyceroporins BtEglpA, BtEglpB1, and BtEglpB2. Putative mercury-sensitive cysteines are shown in bold pink text. BtDrip2 has three variants, differing in the position of the Met start site (denoted in bold red text). Schematic beneath sequence alignment shows predicted domain structure of BtAqps. Rectangular boxes denote the position of the transmembrane helices (blue boxes) and the hemi-helices with the NPA motifs (green boxes). Intra- and extracellular loops A-E are denoted as ovals with letters. $\mathrm{N}^{\mathrm{t}}$ : amino-terminal domain, $\mathrm{C}^{\mathrm{t}}$ : carboxylterminal domain.

Figure 2. Phylogenetic analysis and classification of hemipteran aquaporins. Bayesian majority-rule consensus tree of aquaporins from 11 superfamilies of hemipteran insects. The tree is mid-point rooted and inferred from 750,000 MCMC generations of a codon alignment of 114 non-redundant aquaporins. Posterior probabilities of the codon/amino acid analyses are shown at each node, with “_" representing a polytomy $(<50 \%)$. The scale bar indicates the rate of nucleotide substitution per site.

Figure 3. Spatio-temporal transcription profiles of Bemisia tabaci aquaporins. (A) Reverse transcriptase-PCR transcriptional expression of BtAqps in B. tabaci developmental stages. cDNA was prepared from total RNA obtained from pools of eggs, $1^{\text {st }}$ instars, $2^{\text {nd }}-3^{\text {rd }}$ instars, $4^{\text {th }}$ instars, and adults. (B) Transcriptional expression profile of BtAqps from adult tissues. cDNA was prepared from total RNA from dissected guts, whole bodies minus guts, head/thoraces, and abdomens. BtRpl29 was amplified as an internal reference. Aquaporins and reference gene were amplified for 30 and 35 cycles, electrophoresed on 2\% agarose gels and visualized using SYBR Safe (Invitrogen).

Figure 4. Cellular localization of Bemisia tabaci aquaporins heterologously produced in cultured Trichoplusia ni cells. Recombinant B. tabaci aquaporins were expressed in $T$. $n i$ cells as protein chimeras with the enhanced green fluorescent protein (EGFP) at their carboxyl-termini. Top image of each panel shows fluorescence detection and the bottom image corresponds to the same image under phase contrast. Images were captured using a Fluoview FV10i-LIV laser scanning confocal microscope using a 60x phase contrast water-immersion objective (NA 1.2) at 102x amplification. (A) EGFP control, (B) BtDrip1-EGFP chimera, (C) BtDrip2 variant 1-EGFP chimera, (D) BtDrip2 variant 2-EGFP chimera, (E) BtDrip2 variant 3-EGFP chimera, $(\mathrm{F})$ BtPrip-EGFP chimera, $(\mathrm{G})$ BtEglpA-EGFP chimera, (H) BtEglpB1-EGFP chimera, (I) BtEglpB2-EGFP chimera, (J) BtAqp12L-EGFP chimera. Scale bar $=10 \mu \mathrm{m}$ in all panels.

Figure 5. Osmotic water permeability and glycerol uptake of Xenopus laevis oocytes expressing Bemisia tabaci aquaporins. (A) Osmotic water permeability and (B) ${ }^{3} \mathrm{H}$ radiolabled glycerol uptake of $X$. laevis oocytes injected with water (control) or expressing BtAqps (BtDrip1, BtDrip2v1, BtDrip2v2, BtDrip2v3, BtPrip, BtEglpA, 
BtEglpB1, BtEglpB2, BtAqp12L) or human AQP1 and AQP3 (hAQP1 and hAQP3, respectively). Student's $t$-test was performed to find means that are significantly ( $p<$ 0.05 ) different from the water-injected control oocytes (indicated with asterisks). Box plots show $25^{\text {th }}$ and $75^{\text {th }}$ quartiles (box), mean values (solid vertical line within box), and error bars span the full range of data points. Sample sizes are $(n)$ are shown at right of plot for each sample.

\section{Acknowledgments}

We thank Lynn Forlow-Jech, Sarah Bjorklund, and Dannialle LeRoy for technical support. This is a cooperative investigation between USDA-ARS and the Institut de Recerca I Tecnologia Agroalimentaries (IRTA). Mention of trade names or commercial products in this article is solely for the purpose of providing specific information and does not imply recommendation or endorsement by the U. S. Department of Agriculture. USDA is an equal opportunity provider and employer.

\section{Funding}

This work was supported by base CRIS funding to USDA-ARS, National Program 304 Crop Protection \& Quarantine [Project \#2020-22620-022-00D] to J.F., and partially funded by the Research Council of Norway Grants 204813/F20 and 254872/E40 to R.N.F. and the Spanish Ministry of Economy and Competitiveness (MINECO), project AGL2013-41196-R to J.C. E.V.E. was supported by the USDA-ARS Research Associate Program to J.F.

\section{References}

Abascal, F., Irisarri, I., Zardoya, R., 2014. Diversity and evolution of membrane intrinsic proteins. Biochim. Biophys. Acta. 1840, 1468-1481.

Agre, P., Preston, G. M., Smith, B. L., Jung, J. S., Raina, S., Moon, C., Guggino, W. B. and Nielson, S., 1993. Aquaporin CHIP: the archetypal molecular water channel. Am. J. Physiol. 265, F463-F476. 
Amiry-Moghaddam, M., Lindland, H., Zelenin, S., Roberg, B., Gundersen, B.B., Petersen, P., Rinvik, E., Torgner, I.A., Ottersen, O.P., 2005. Brain mitochondria contain aquaporin water channels: evidence for the expression of a short AQP9 isoform in the inner mitochondrial membrane. FASEB J. 19, 1459-1467.

Beitz, E., Wu, B., Holm, L.M., Schultz, J.E., Zeuthen, T., 2006. Point mutations in the aromatic/arginine region in aquaporin 1 allow passage of urea, glycerol, ammonia, and protons. Proc. Natl. Acad. Sci. USA. 103, 269-274.

Benga, G., 2009. Water channel proteins (later called aquaporins) and relatives: past, present, and future. IUBMB Life. 61, 112-133.

Bernsel, A., Viklund, H., Hennerdal, A., Elofsson, A., 2009. TOPCONS: consensus prediction of membrane protein topology. Nucleic Acids Res. 37, W465-W468.

Blom, N., Gammeltoft, S., Brunak, S., 1999. Sequence- and structure-based prediction of eukaryotic protein phosphorylation sites. J. Mol. Biol. 294, 1351-1362.

Byrne, D.N., Bellows, T.S. Jr., 1991. Whitefly biology. Annu. Rev. Entomol. 36, 431457.

Campbell, E.M., Ball, A., Hoppler, S., Bowman, A.S., 2008. Invertebrate aquaporins: a review. J. Comp. Physiol. B. 178, 935-955.

Cicero, J.M., Hiebert, E., Webb, S.E., 1995. The alimentary canal of Bemisia tabaci and Trialeurodes abutilonea (Homoptera, Sternorrhynchi): histology, ultrastructure and correlations to function. Zoomorphology. 115, 31-39.

Clausen, H., 2013. Precision mapping of the human O-GalNAc glycoproteome through SimpleCell technology. EMBO J. 32, 1478-1488.

Cohen, E., 2012. Roles of aquaporins in osmoregulation, desiccation and cold hardiness in insects. Entomol. Ornithol. Herpetol. S1, 001.

Cohen, E., 2013. Chapter one - water homeostasis and osmoregulation as targets in the control of insect pests. In: Cohen, E. (Ed.), Target Receptors in the Control of Insect Pests: Part I, Advances in Insect Physiology, vol. 44. Academic Press, pp. 1-61.

Cosson, P., Letourneur, F., 1994. Coatomer interaction with di-lysine endoplasmic reticulum retention motifs. Science. 263, 1629-1631.

Douglas, A.E., 2006. Phloem-sap feeding by animals: problems and solutions. J. exp. Bot. 57, 747-754.

Drake, L.L., Boudko, D.Y., Marinotti, O., Carpenter, V.K., Dawe, A.L., Hansen, I.A., 2010. The aquaporin gene family of the yellow fever mosquito, Aedes aegypti. PLoS ONE. 5, e15578. 
Drake, L.L., Rodriguez, S.D., Hansen, I.A., 2015. Functional characterization of aquaporins and aquaglyceroporins of the yellow fever mosquito, Aedes aegypti. Scientific Reports. 5, 7795.

Duchesne, L., Hubert, J.F., Verbavatz, J.M., Thomas, D., Pietrantonio, P.V., 2003. Mosquito (Aedes aegypti) aquaporin, present in tracheolar cells, transports water, not glycerol, and forms orthogonal arrays in Xenopus oocyte membranes. Eur. J. Biochem. $270,422-429$.

Fabrick, J.A., Pei, J., Hull, J.J., Yool, A.J., 2014. Molecular and functional characterization of multiple aquaporin water channel proteins from the western tarnished plant bug, Lygus hesperus. Insect Biochem. Molec. Biol. 45, 125-140.

Finn, R.N., Cerdà, J., 2015. Evolution and functional diversity of aquaporins. Biol. Bull. 229, 6-23.

Finn, R.N., Chauvigné, F., Hlidberg, J.B., Cutler, C.P., Cerdà, J., 2014. The lineagespecific evolution of aquaporin gene clusters facilitated tetrapod terrestrial adaptation. PLoS ONE. 9, e113686.

Finn, R.N., Chauvigné, F., Stavang, J.A., Belles, X., Cerdà, J., 2015. Insect glycerol transporters evolved by functional co-option and gene replacement. Nature Comm. 6 , 7814.

Frank, K., Sippl, M.J., 2008. High performance signal peptide prediction based on sequence alignment techniques. Bioinformatics. 24, 2172-2176.

Gomes, D., Agasse, A. Thiébaud, P., Delrot, S., Gerós, H., Chaumont, F., 2009. Aquaporins are multifunctional water and solute transporters highly divergent in living organisms. Biochimica et Biophysica Acta. 1788, 1213-1228.

Gonen, T., Walz, T., 2006. The structure of aquaporins. Q. Rev. Biophys. 39, 361-396.

Goodchild, A.J.P., 1966. Evolution of the alimentary canal in the Hemiptera. Biol. Rev. 41, 97-139.

Gorelick, D.A., Praetorius, J., Tsunenari, T., Nielsen, S., Agre, P., 2006. Aquaporin11: A channel protein lacking apparent transport function expressed in brain. BMC Biochem. 7:14.

Goto, S.G., Lee, R.E., Denlinger, D.L., 2015. Aquaporins in the Antarctic midge, an extremophile that relies on dehydration for cold survival. Biol. Bull. 229, 47-57.

Goto, S.G., Philip, B.N., Teets, N.M., Kawarasaki, Y., Lee Jr., R.E., Denlinger, D.L., 2011. Functional characterization of an aquaporin in the Antarctic midge Belgica antarctica. J. Insect Physiol. 57, 1106-1114.

Han, M.V., Zmasek, C.M., 2009. phyloXML: XML for evolutionary biology and comparative genomics. BMC Bioinformatics. 10, 356. 
Herraiz, A., Chauvigne, F., Cerdà, J., Bellas, X., Piulachs, M.-D., 2011. Identification and functional characterization of an ovarian aquaporin from the cockroach Blattella germanica L. (Dictyoptera, Blattellidae). J. Exp. Biol. 214, 3630-3638.

Hofmann, K., Stoffel, W., 1993. TMbase e a database of membrane spanning proteins segments. Biol. Chem. Hoppe-Seyler 374, 166.

Horton, P., Park, K.J., Obayashi, T., Fujita, N., Harada, H., Adams-Collier, C.J., Nakai, K. 2007. WoLF PSORT: protein localization predictor. Nucleic Acids Res. 35, W585587.

Hubert, J.F., Thomas, D., Cavalier, A., Gouranton, F., 1989. Structural and biochemical observations on specialized membranes of the filter chamber, a watershunting complex in sap-sucking homopteran insects. Biol. Cell. 66, 155-163.

Ibanez, F., Hancock, J., Tamborindeguy, C., 2014. Identification and expression analysis of aquaporins in the potato psyllid, Bactericera cockerelli. PLoS ONE. 9, e111745.

Ishibashi, K., 2006. Aquaporin subfamily with unusual NPA boxes. Biochim. Biophys. Acta. 1758, 989-993.

Jing, X., White, T.A., Luan, J., Jiao, C., Fei, Z., Douglas, A.E., 2016. Evolutionary conservation of candidate osmoregulation genes in plant phloem sap-feeding insects. Insect Mol. Biol. 25, 351-358.

Jung, J.S., Preston, G.M., Smith, B.L., Guggino, W.B., Agre, P., 1994. Molecular structure of the water channel through aquaporin CHIP. The hourglass model. J. Biol. Chem. $269,14648-14654$.

Käll, L., Krogh, A., Sonnhammer, E.L.L., 2004. A combined transmembrane topology and signal peptide prediction method. J. Mol. Biol. 338, 1027-1036.

Kambara, K., Takematsu, Y., Azuma, M., Kobayashi, J., 2009. cDNA cloning of aquaporin gene expressed in the digestive tract of the Formosan subterranean termite, Coptotermes formosanus shiraki (Isoptera; Rhinotermitidae). Appl. Entomol. Zool. 44, 315-321.

Kataoka, N., Miyake, S., Azuma, M., 2009a. Aquaporin and aquaglyceroporin in silkworms, differently expressed in the hindgut and midgut of Bombyx mori. Insect Mol. Biol. 18, 303-314.

Kataoka, N., Miyake, S., Azuma, M., 2009b. Molecular characterization of aquaporin and aquaglyceroporin in the alimentary canal of Grapholita molesta (the oriental fruit moth) comparison with Bombyx mori aquaporins. J. Insect Biotechnol. 78, 81-90.

Katoh, K., Toh, H., 2008. Recent developments in the MAFFT multiple sequence alignment program. Brief Bioinform. 9, 286-298. 
Kaufmann, N., Mathai, J.C., Hill, W.G., Dow, J.A.T., Zeidel, M.L., Brodsky, J.L., 2005. Developmental expression and biophysical characterization of a Drosophila melanogaster aquaporin. Am. J. Physiol. Cell Physiol. 289, C397-C407.

Kikawada, T., Saito, A., Kanamori, Y., Fujita, M., Snigorska, K., Watanabe, M., Okuda, T., 2008. Dehydration-inducible changes in expression of two aquaporins in the sleeping chironomid, Polypedilum vanderplanki. BBA-Biomemb. 1778, 514-520.

King, L.S., Kozono, D., Agre, P., 2004. From structure to disease: the evolving tale of aquaporin biology. Nature Rev. Mol. Cell Biol. 5, 687-698.

Krogh, A., Larsson, B., von Heijne, G., Sonnhammer, E.L.L., 2001. Predicting transmembrane protein topology with a hidden Markov model: application to complete genomes. J. Mol. Biol. 305, 567-580.

Le Cahérec, F., Guillam, M.T., Beuron, F., Cavalier, A., Thomas, D., Gouranton, J., Hubert, J.F., 1997. Aquaporin-related proteins in the filter chamber of homopteran insects. Cell Tiss. Res. 290, 143-151.

Lee, K.S., Kim, S.R., Lee, S.M., Lee, K.R., Sohn, H.D., Jin, B.R., 2001. Molecular cloning and expression of a cDNA encoding the aquaporin homologue from the firefly, Pyrocoelia rufa. Korean Journal of Entomology. 31, 269-279.

Liu, K., Tsujimoto, H., Cha, S.-J., Agre, P., Rasgon, J.L., 2011. Aquaporin water channel AgAQP1 in the malaria vector mosquito Anopheles gambiae during blood feeding and humidity adaptation. Proc. Natl. Acad. Sci. USA 108, 6062-6066.

Ma, T., Yang, B., Kuo, W.-L., Verkman, A.S., 1996. cDNA cloning and gene structure of a novel water channel expressed exclusively in human kidney: evidence for a gene cluster of aquaporin at chromosome locus 12q13. Genomics, 35, 543-550.

Martini, S.V., Goldenberg, R.C., Fortes, F.S.A., Campos-de-Carvalho, A.C., Falkenstein, D., Morales, M.M., 2004. Rhodnius prolixus Malpighian tubule's aquaporin expression is modulated by 5-hydroxytryptamine. Arch. Insect Biochem. Physiol. 57, 133-141.

Maruyama, M., Kambara, K., Naka, H., Azuma, M., 2015. Insect water-specific aquaporins in developing ovarian follicles of the silk moth Bombyx mori: role in hydration during egg maturation. Biol. Bull. 229, 58-69.

Mathew, L.G., Campbell, E.M., Yool, A.J., Fabrick, J.A., 2011. Identification and characterization of functional aquaporin water channel protein from alimentary tract of whitefly, Bemisia tabaci. Insect Biochem. Mol. Biol. 41, 178-190.

Moe, S.E., Sorbo, J.G., Sogaard, R., Zeuthen, T., Ottersen, O.P., Holen, T., 2008. New isoforms of rat aquaporin-4. Genomics. 91, 367-377. 
Morishita, Y., Matsuzaki, T., Hara-chikuma, M., Andoo, A., Shimono, M., Matsuki, A., Kobayashi, K., Ikeda, M., Yamamoto, T., Verkman, A., Kusano, E., Ookawara, S., Takata, K., Sasaki, S., Ishibashi, K., 2005. Disruption of aquaporin-11 produces polycystic kidneys following vacuolization of the proximal tubule. Mol. Cell.

Biol. 25, 7770-7779.

Murata, K., Mitsuoka, K, Hirai, T., Walz, T. Agre, P., Heymann, J.B., Engel, A., Fujiyoshi, Y., 2000. Structural determinants of water permeation through aquaporin-1. Nature. 407, 599-605.

Nagae, T., Miyake, S., Kosaki, S., Azuma, M., 2013. Identification and characterization of a functional aquaporin water channel (Anomala cuprea DRIP) in a coleopteran insect. J. Exp. Biol. 216, 2564-2572.

Navas-Castillo, J., Fiallo-Olivé, E., Sánchez-Campos, S., 2011. Emerging virus diseases transmitted by whiteflies. Annu. Rev. Phytopathol. 49, 219-248.

Philip, B.N., Kiss, A.J., Lee Jr., R.E., 2011. The protective role of aquaporins in the freeze-tolerant insect Eurosta solidaginis: functional characterization and tissue abundance of EsAQP1. J. Exp. Biol. 214, 848-857.

Raza, A., Malik, H.J., Shafiq, M., Amin, I., Scheffler, J.A., Scheffler, B.E., Mansoor, S., 2016. RNA interference based approach to down regulate osmoregulators of whitefly (Bemisia tabaci): Potential technology for the control of whitefly. PLoS ONE. $11, \mathrm{e} 0153883$.

Ronquist, F., Huelsenbeck, J. P., 2003. MrBayes 3: Bayesian phylogenetic inference under mixed models. Bioinformatics. 19, 1572-1574.

Rose, A., Lorenzen, S., Goede, A., Gruening, B., Hildebrand, P.W., 2009. RHYTHMserver to predict the orientation of transmembrane helices in channels and membrane-coils. Nucleic Acids Res. 37, W575-W580.

Salvucci, M.E., 2003. Distinct sucrose isomerases catalyze trehalulose synthesis in whiteflies, Bemisia argentifolii, and Erwinia rhapontici. Comp. Biochem. Physiol. Part B. $135,385-395$.

Shakesby, A.J., Wallace, I.S., Isaacs, H.V., Pritchard, J., Roberts, D.M., Douglas, A.E., 2009. A water-specific aquaporin involved in aphid osmoregulation. Insect Biochem. Mol. Biol. 39, 1-10.

Spring, J.H., Robichaux, S.R., Hamlin, J.A., 2009. The role of aquaporins in excretion in insects. J. Exp. Biol. 212, 358-362.

Staniscuaski, F., Paluzzi, J.-P., Real-Guerra, R., Carlini, C.R., Orchard, I., 2013. Expression analysis and molecular characterization of aquaporins in Rhodnius prolixus. J. Insect Physiol. 59, 1140-1150. 
Stavang, J.A., Chauvigné, F., Kongshaug, H., Cerdà, J., Nilsen, F., Finn, R.N., 2015. Phylogenomic and functional analyses of salmon lice aquaporins uncover the molecular diversity of the superfamily in Arthropoda. BMC Genomics. 16, 618.

Steentoft, C., Vakhrushev, S.Y., Joshi, H.J., Kong, Y., Vester-Christensen, M.B., Schjoldager, K.T., Lavrsen, K., Dabelsteen, S., Pedersen, N.B., Marcos-Silva, L., Gupta, R., Bennett, E.P., Mandel, U., Brunak, S., Wandall, H.H., Levery, S.B., Sui, H., Han, B.G., Lee, J.K., Walian, P., Jap, B.K., 2001. Structural basis of waterspecific transport through the AQP1 water channel. Nature. 414, 872-878.

Suyama, M., Torrents, D., Bork, P., 2006. PAL2NAL: robust conversion of protein sequence alignments into the corresponding codon alignments. Nucleic Acids Res. 34, W609-W612.

Törnroth-Horsefield, S., Hedfalk, K., Fischer, G., Lindkvist-Petersson, K., Neutze, R., 2010. Structural insights into eukaryotic aquaporin regulation. FEBS Letters. 584, 2580-2588.

Tsujimoto, H., Liu, K., Linser, P., Agre, P., Rasgon, J.L., 2013. Organ-specific splice variants of aquaporin water channel AgAQP1 in the malaria vector Anopheles gambiae. PLoS ONE. 8, e75888.

Tusnady, G.E., Simon, I., 2001. The HMMTOP transmembrane topology prediction server. Bioinformatics. 17, 849-850.

Tzin, V., Yang, X., Jing, X., Zhang, K., Jander, G., Douglas, A.E., 2015. RNA interference against gut osmoregulatory genes in phloem-feeding insects. J. Insect Physiol. 79, 105-112.

Wallace, I.S., Shakesby, A.J., Hwang, J.H., Choi, W.G., Martinkova, N., Douglas, A.E., Roberts, D.M., 2012. Acyrthosiphon pisum AQP2: a multifunctional insect aquaglyceroporin. Biochim. Biophys. Acta. 1818, 627-635.

Wang, X.W., Luan, J.B., Li, J.M., Bao, Y.Y., Zhang, C.X., Liu, S.S., 2010. De novo characterization of a whitefly transcriptome and analysis of its gene expression during development. BMC Genomics. 11, 400.

Wurch, T., Lestienne, F. and Pauwels, P., 1998. A modified overlap extension PCR method to create chimeric genes in the absence of restriction enzymes. Biotechnol. Tech. $12,653-657$.

Xie, W., Meng, Q.S., Wu, Q.J., Wang, S.L., Yang, X., Yang, N.N., Li, R.M., Jiao, X.G., Pan, H.P., Liu, B.M., Qi, S., Xu, B.Y., Hu, S.N., Zhou, X.G., Zhang, Y.J., 2012. Pyrosequencing the Bemisia tabaci transcriptome reveals a highly diverse bacterial community and a robust system for insecticide resistance. PLoS ONE. 7, e35181.

Yi, S.-X., Benoit, J.B., Elnitsky, M.A., Kaufmann, N., Brodsky, J.L., Zeidel, M.L., Denlinger, D.L., Lee, R.L., 2011. Function and immuno-localization of aquaporins in the Antarctic midge Belgica antarctica. J. Insect Physiol. 57, 1096-1105. 
Yool, A.J., Weinstein, A.M., 2002. New roles for old holes: ion channel function in aquaporin-1. News Physiol. Sci. 17, 68-72.

Zardoya, R., 2005. Phylogeny and evolution of the major intrinsic protein family. Biol. Cell. 97, 397-414. 


\section{Figure 1 \\ BtDripl BtDrip2 Btprip BtEglpA BtEglpB1 BtEglpB? BEAqp 12L}

BtDrip1 BtDrip2 BtPrip BtEglpA BtEg $1 \mathrm{pB} 1$ BtEglpB? Bt.Aqp 12L

BtDrip1 BtDrip2 BtPrip BtEgIPA BtEglpa1 BtEgIpB2 BLAqP 12L

BtDrip1 BtDrip2 BtPrip BtEglpA BtEglpB1 BtEglpB2 Bt:Aqp 12L

BtDrip1 BtDrip2 BtPrip BtEglpA BtEglpa1 BtEglpB2 BEAqP 12L

BtDrip1 BtDrip2 BtPrip BtEglpA BtEg1pB1 BtEglpB? BtAqp 12L

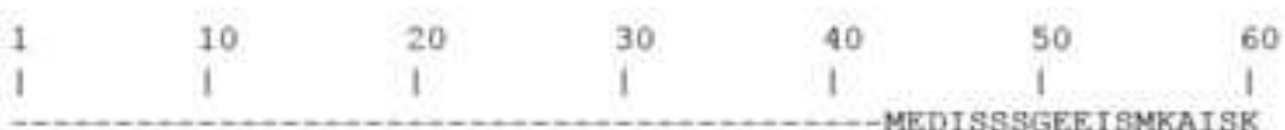
MKQSHPLHCSELVQFPRERSVRCVAAPESAYLPSERLELPHPLPAASEKQPNKMSAVISR MAASLKA MPFQRDPPNSVFEP MSIR-D-.-GAFTP -MGTASVFGLAVSTSYIF

\section{$\mathrm{N}^{\mathrm{t}}$}

VIGVPDIRDGPT-LTKCIVAEFVGTLLLVLIGCMS-VAFVHQDWFVDVVKIAMA VIGVPDVKNWEM-LTRCLIAEFIGLLLLVLIGCMS-IAFSKTDNFLDVVKIALA FLIIA PLGVQEVTDVKSGLGKALVAEALGTLEINFFGCLSAVNLVEPQAAPNLVLIALT FFIVF

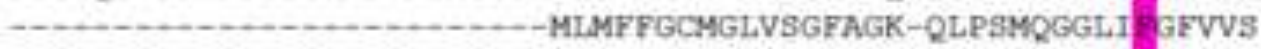
HEFKNLWTKKVWLLLAKGLAELCGTAILLSIGCMSLISNSAGG-VQDHLSVVIS PFAIA HEFPNLDKEKAWFLLEKGLAEMFGTGLFVCMGCMGTLSDSKGE-YLDYLGGVLS AFAIA LTSLIAFWIRKFIHRILNRDTLLRSVLLEFVAAAELCASCEEIAIVADNYGILALAVYLF

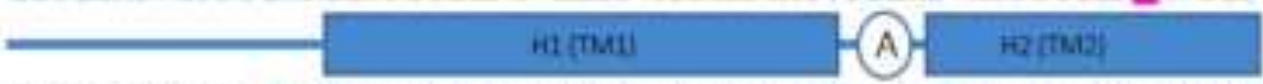
SMVQAIGHVSG-CHINPAVICGLAVSGHVSIIKGMLYIVAQCLGAICGAI ILNEITPKTG TMVQSIGHVSG-CHINPAVTCGLAISGHISLIKAVLYIVVQCLGAVCGAI ILSEISPSSG VAVQSVGHVSG-AHINPAITVGLIVFGKVFI IPAFLYIVVQVLGAIAGSAILRALTPSSTIICIFGHIST-AHLNPAVZAASVVLGNTTPFAVVYLLSECIGACLGEGLLKEITPSDI TSIIIFGHISG-SHINPAITAADVVAGEKSIFMFIYYTVMQCFGAFVGYSILRVLVPNEV TSVMI FGHIS - CI INPALVAADVIRGEKSLFMFVYITVMECLGAFLGYALLAVLVADDL SLTIWWGYSWGSATACPYTHLEDVIEGRTDLRVALLICWAEI GGGLAIFRY INYLWSLEI

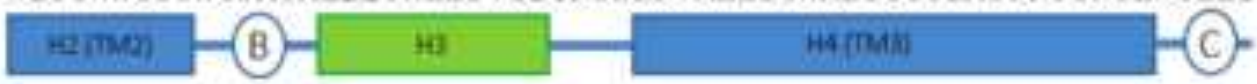
YTAA---GNLGVTTLSTGVS-DLQGVAIEALITFVLLLVVQSVCDGKRTDIKGSIGVAI FGKG---GNLGVTTVSAELS-AKDGFIVEAIITFVLILVVQSVCDVRRTDLKGSVGVAI --YD---GGEGVNKLANNLT-PIQGLGIEEILGEILVLVVFGVCDANRQHTQTIAALTI FDFGVDPAVGLCSTVPHPSLTHSWOAFMGEVLATSFLI FLVCSVWDAPNADKSDSVALKF LGE--KEAGFCCKAVDPRMS-TAQAVGVEMLITGILSLVFCAVQDTKNPDAGQTVAIKF LGR-DSGSFCCTAKDVRVT-SLRAMGVEMLITAILCLLFCSVQDPKNSDPNSTVALKF ASVH-- KGRAWACTADLQVHMILGALVEAGATCLCRLFSRILGDLEPKFSWYIDAFE

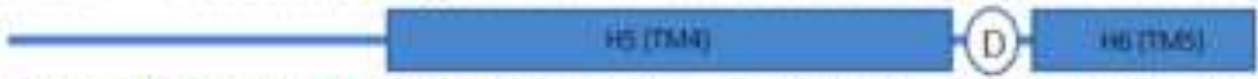
GFAIACCHLAAIKYI ASMNPAPSLGPAFVSG---IWDKHWVYWAGPILGGVTASLLYAI GFAITCCHLAAIKYT ASMIPARSFGPALVSG---IWDNHWVYWAGPILGGVVAGLIYTG GLTVAAGHLATIDF" FGMNPAPSFGAAVIES---FWENHWVFWVPILGGIAASLLYTY ALTIAAISIAEGPYT ASMAPARTLAPAIFNA---VWTMHWVYWVAPISSGIITAFIYKH GVVCVAI IIVPAAHFI ISMNPAPSFGPALFSR---NWKDHWVYWI GPMSGAMIAAYVYTH GFGCFALLLPSVHYR SSMMPARSFGPALYNN---HWKDOWIYWVGEMSGAIISAVIYML. G-DSIEVAAFNS SYFNPVIAATALKYGEGNTTIEHFVVYWIGACLGSVAAITFYNR

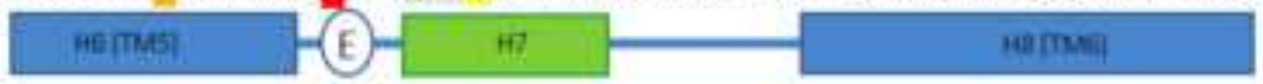

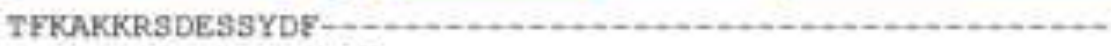

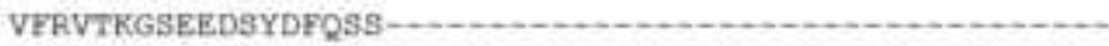

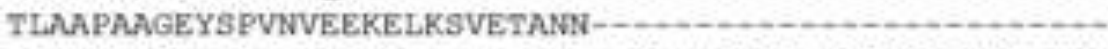

IFNRKRRTSMDEPIQSIDDINAEVPLTGVISSKHPSIEKIQDSPLTKRKANNS LFARHHPSKSRSVSR - - QPSARITLQHQQNHSGDEV - _...........

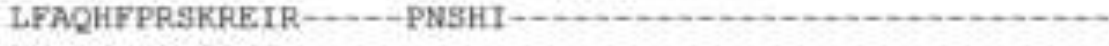

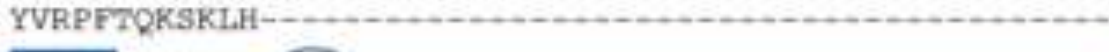

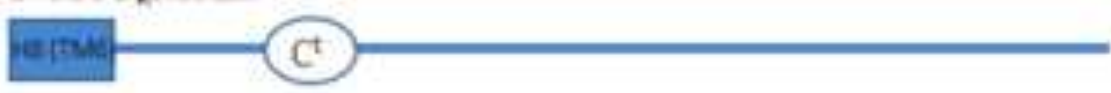


- Aleyrodoidea

I Coccoidea

Aphidoidea

$100 / 100^{2}$ Diaphorina citri Bib

Psylloidea

Reduvioidea

Cimicoidea

Lygaeoidea

Pentatomoidea

Aleyrodoidea

Psylloidea

Membracoidea

Fulgoroidea

Aphidoidea

Coccoidea

Cimicoidea

Reduvioidea

Pentatomoidea

Lygaeoidea

Gerroidea

Coccoidea

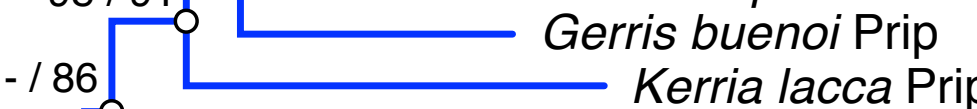
lectularius Prip

Psylloidea

Membracoidea

Fulgoroidea

- Aleyrodoidea

$55 / 98$ $\longrightarrow$ Bemisia tabaci Prip

Cimicoidea

Reduvioidea

Lygaeoidea

Gerroidea

Lygus hesperus EglpA (NT_v1-5)

Rhodnius prolixus EglpA

Rhodnius neglectus EglpA

\section{Gerris buenoi EglpA
Graminella nigrifrons EglpA}

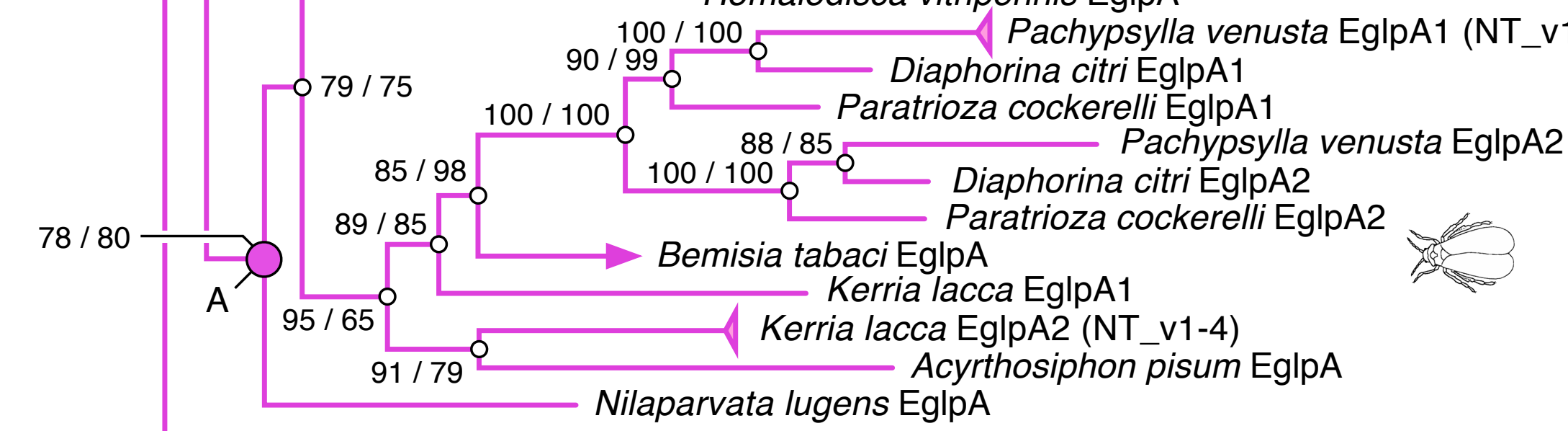

Pachypsylla venusta EglpA1 (NT_v1-2)

Membracoidea

Psylloidea

EglpA

$100 / 100$

$70 / 65$

$100 / 100$ Rhodnius prolixus EglpB

$88 / 98$ Oncopeltus fasciatus Eglp
Halyomorpha halys EglpB

\section{Rhodnius neglectus EglpB}
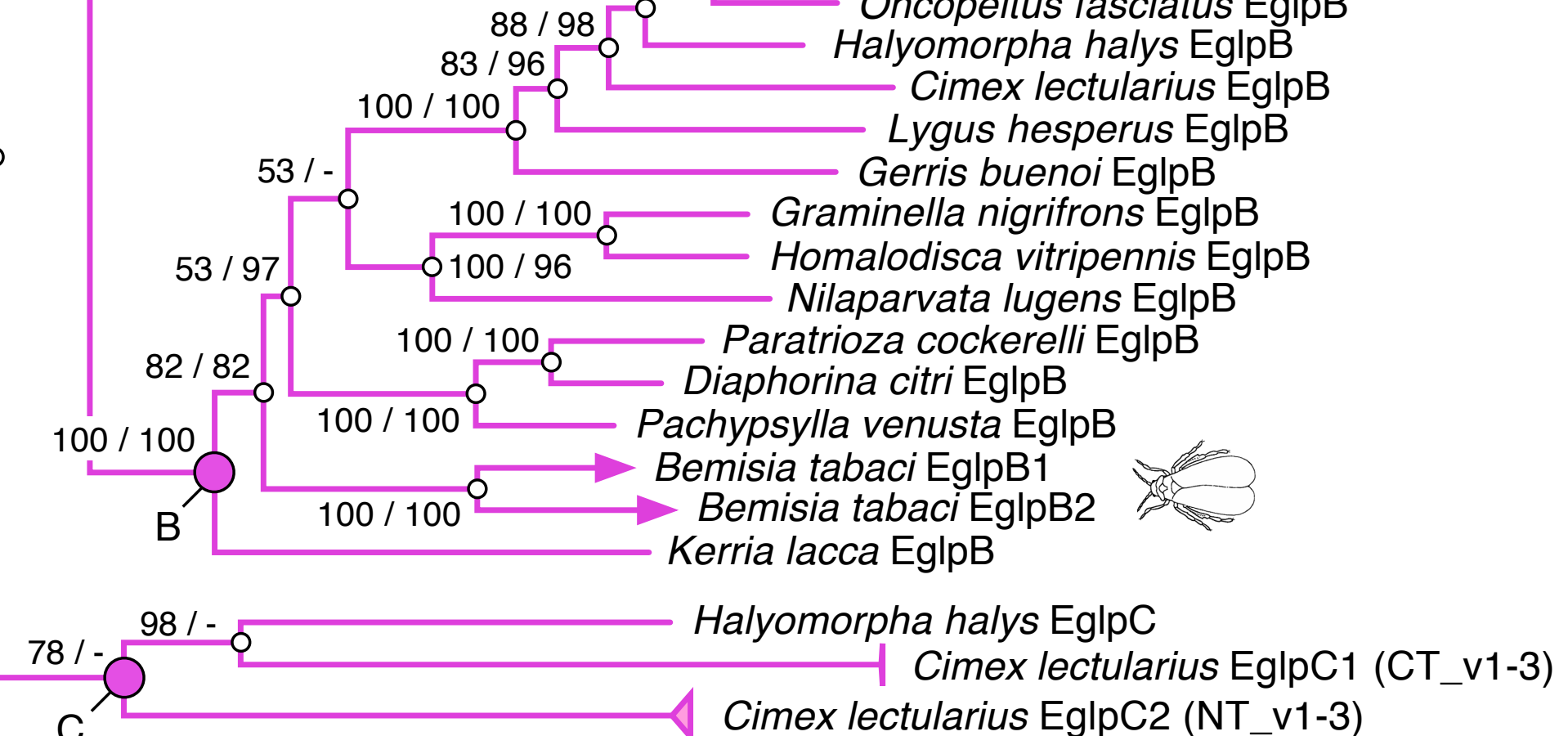

Cimex lectularius EglpC2 (NT_v1-3)

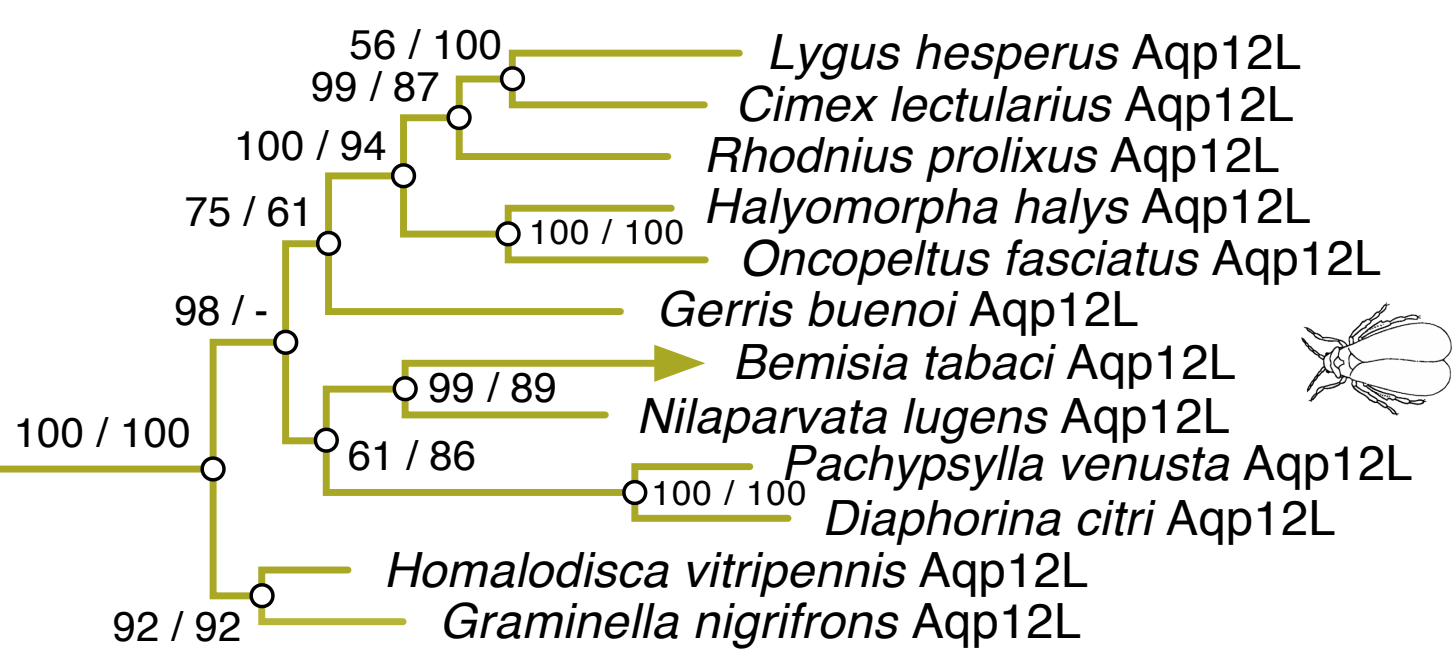

Aleyrodoidea

Coccoidea

Aphidoidea

Fulgoroidea

Reduvioidea

Lygaeoidea

Pentatomoidea

Cimicoidea

Gerroidea

Membracoidea

Fulgoroidea

Psylloidea

Aleyrodoidea

Coccoidea

- Pentatomoidea

Cimicoidea

Cimicoidea

Reduvioidea

Pentatomoidea

Lygaeoidea

Gerroidea

Aleyrodoidea

Fulgoroidea

Psylloidea

Membracoidea
EglpC

EglpB

Aqp12L 
Figure 3

A

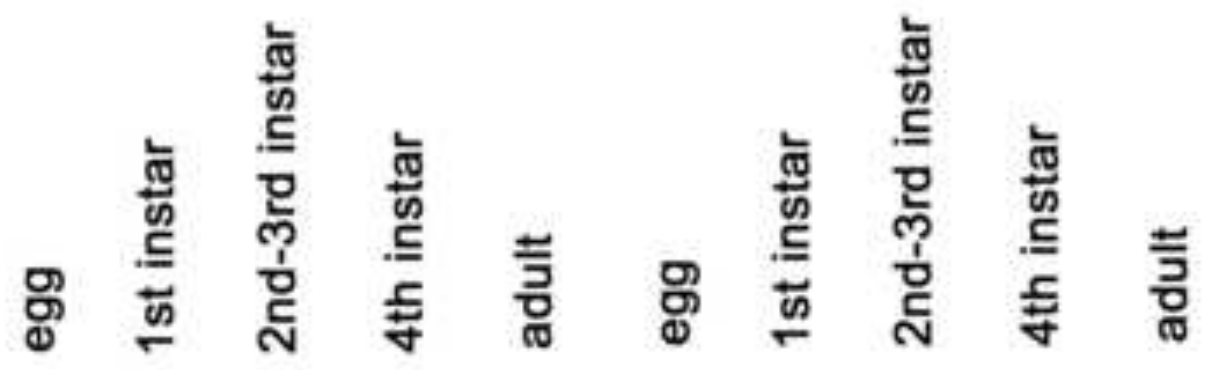

BtDrip1
BtDrip2
BtPrip
BtEglpA
BtEglpB1
BtEglpB2
BtAqp12L
BtRpl29

B

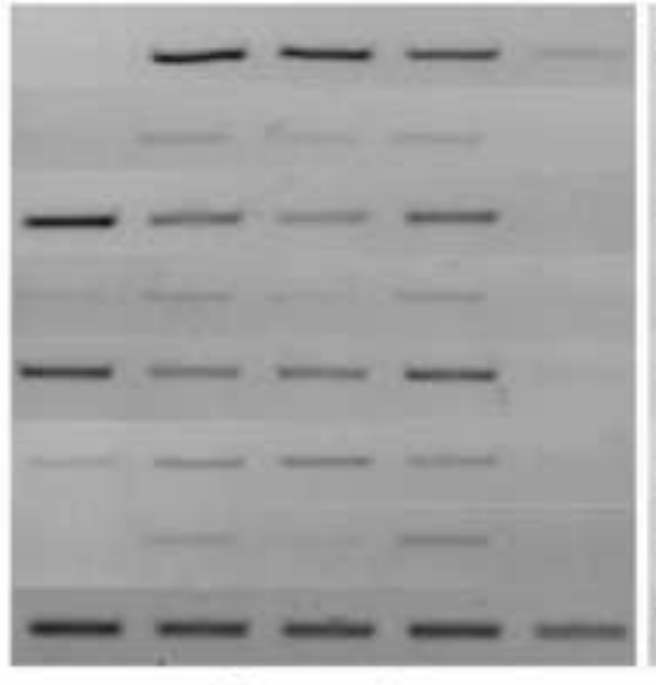

30 cycles

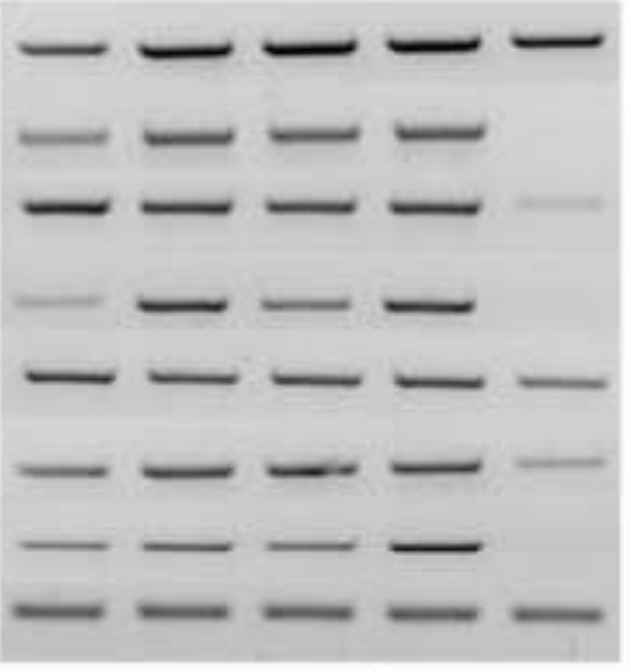

35 cycles
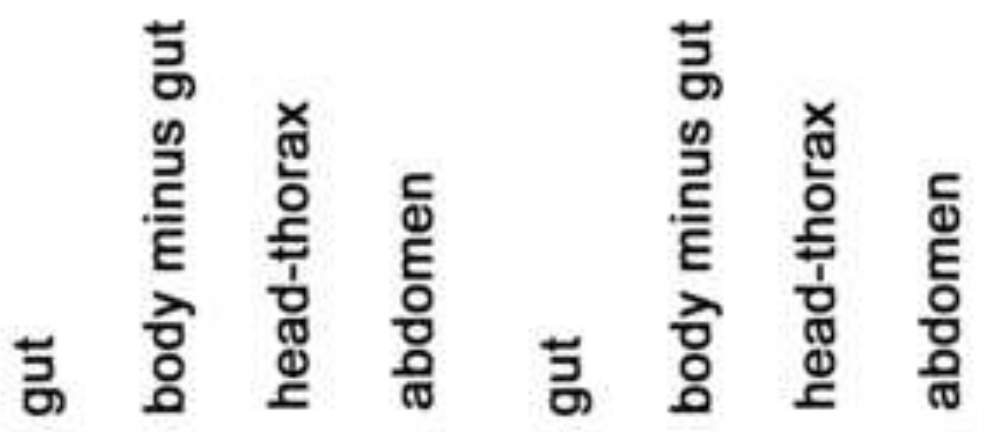
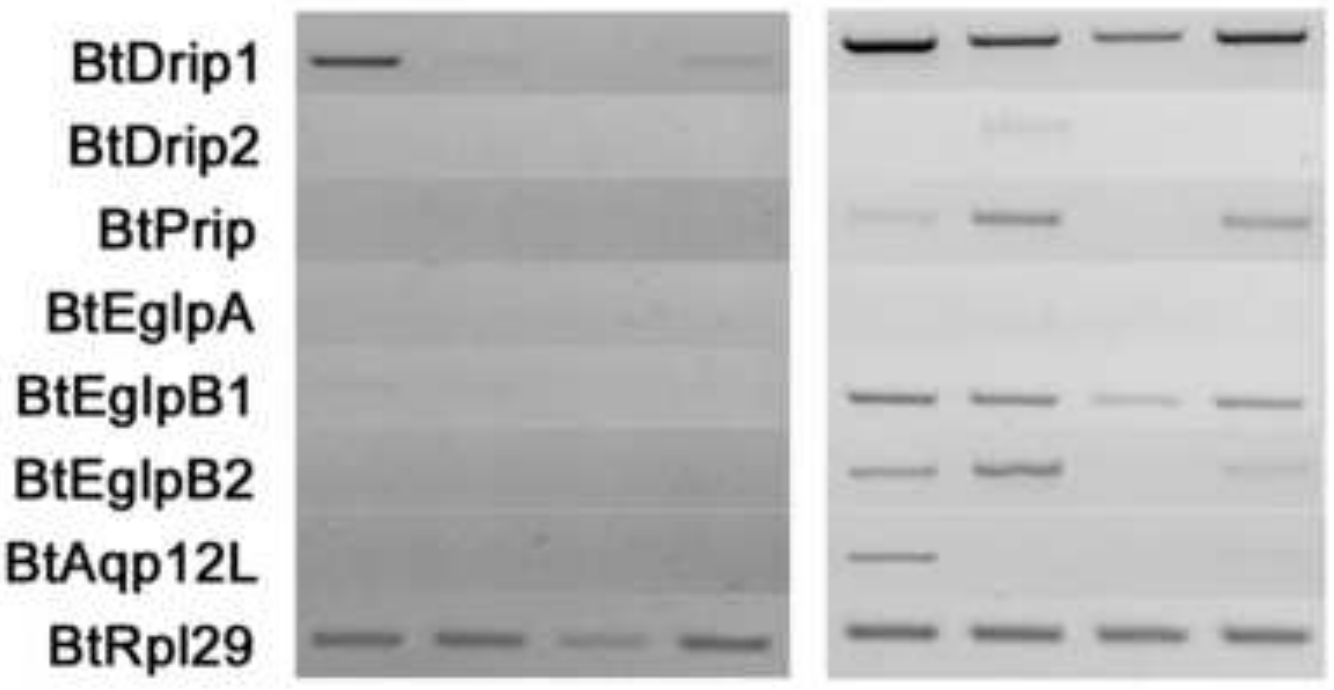

35 cycles 
Figure 5

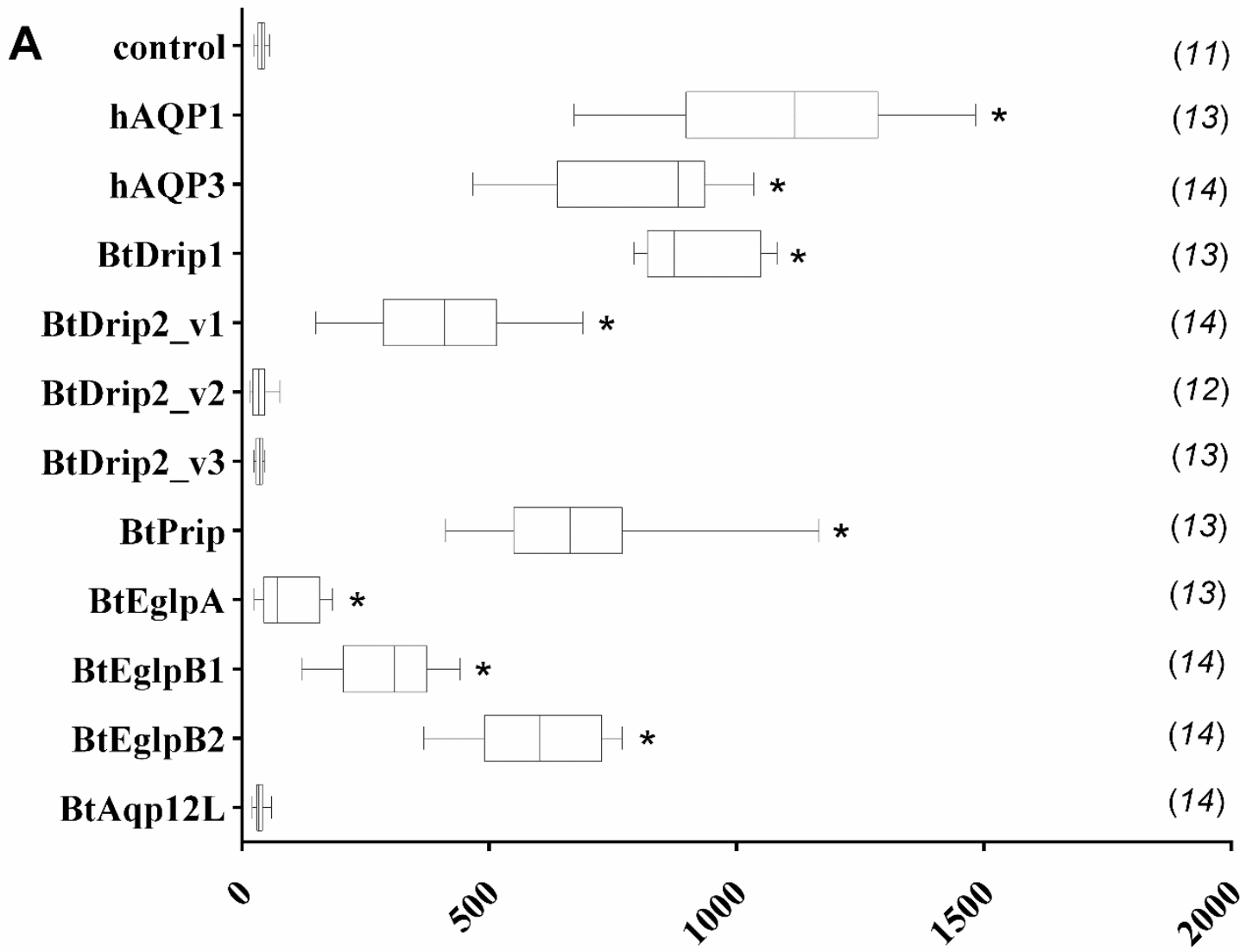

water permeability (Pf) $(\mu \mathrm{m} / \mathrm{s})$

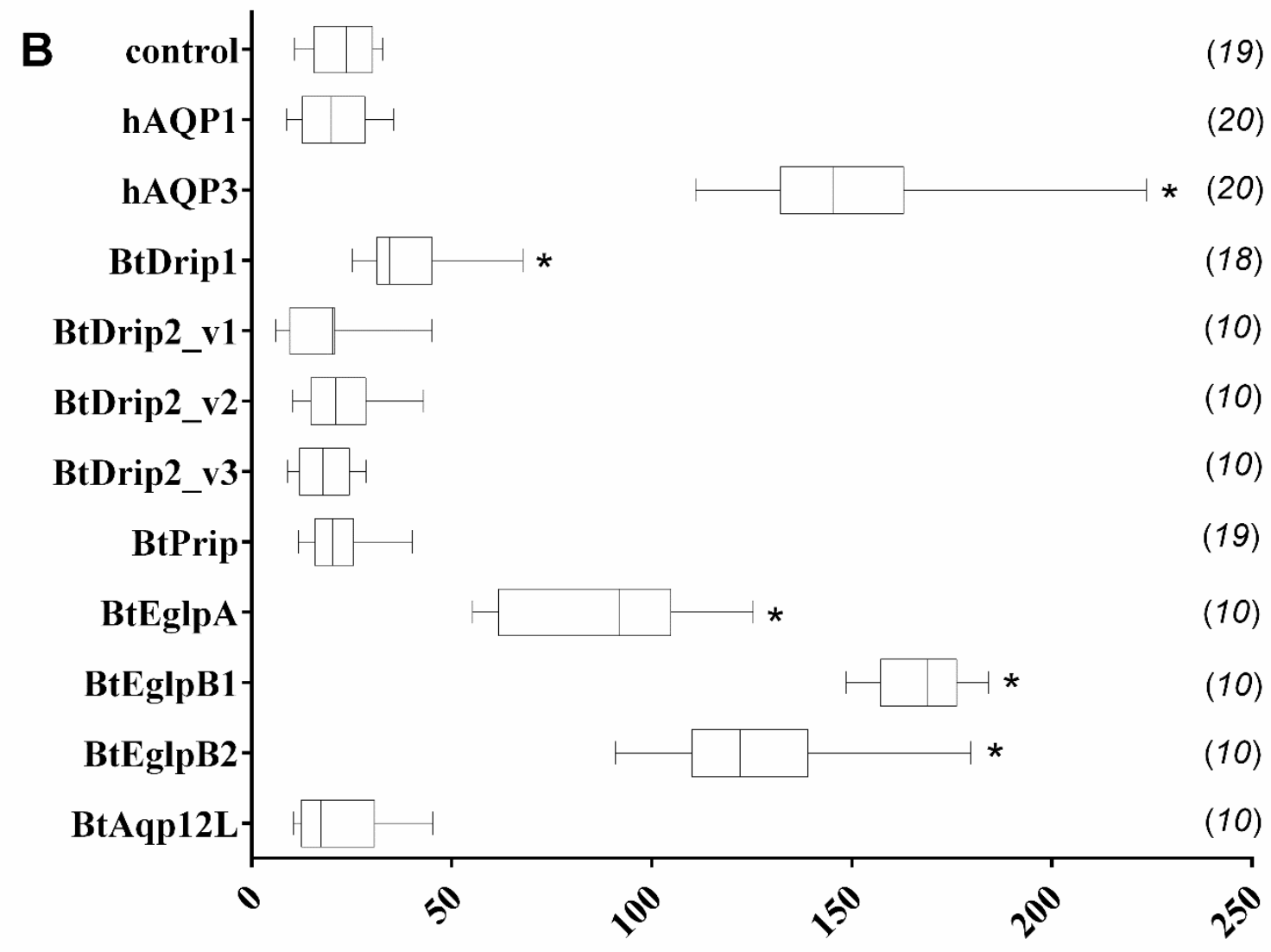

glycerol uptake (pmol/oocyte) 